\title{
JOINT DISTRIBUTIONS FOR MOVEMENTS OF ELEMENTS IN SATTOLO'S AND THE FISHER-YATES ALGORITHM
}

\author{
GUY LOUCHARD, HELMUT PRODINGER, AND STEPHAN WAGNER
}

\begin{abstract}
Sattolo's algorithm creates a random cyclic permutation by interchanging pairs of elements in an appropriate manner; the Fisher-Yates algorithm produces random (not necessarily cyclic) permutations in a very similar way. The distributions of the movements of the elements in these two algorithms have already been treated quite extensively in past works. In this paper, we are interested in the joint distribution of two elements $j$ and $k$; we are able to compute the bivariate generating functions explicitly, although it is quite involved. From it, moments and limiting distributions can be deduced. Furthermore, we compute the probability that elements $i$ and $j$ ever change places in both algorithms.
\end{abstract}

\section{INTRODUCTION}

Sattolo [8] provides an algorithm to generate a random cyclic permutation as follows: starting with the sequence $12 \ldots n$, a random integer between 1 and $n-1$ is chosen, say $i$, and the numbers in positions $i$ and $n$ are interchanged. Then a random integer between 1 and $n-2$ is chosen, say $j$, and the numbers in positions $j$ and $n-1$ are interchanged, and so on. After $n-1$ iterations, a random cyclic permutation is obtained.

Let us give an example, with $n=5$ and the random numbers $4,1,2,1$ :

12354

$5 \underline{2} \mathbf{3} 14$

$\underline{5} \mathbf{3} 214$

35214

The result is the cyclic permutation $1 \rightarrow 3 \rightarrow 2 \rightarrow 5 \rightarrow 4 \rightarrow 1$.

The first treatment of this algorithm has been given in [7] — there, moments of the number of moves that element $k$ makes and the distance that element $k$ travels are given.

In the example we obtain the numbers $1,1,2,1,3$ for the moves and $3,1,2,1,5$ for the distances $(k=1, \ldots, 5)$.

Further developments are provided in papers of Mahmoud and Wilson [4, 9, 10]: Mahmoud determined the limiting distributions for the number of moves as well as for the distances. Assuming that $\frac{k}{n} \rightarrow \alpha$ as $n \rightarrow \infty$, the distribution of the number of moves the element $k$ makes is given by $1+X \cdot \operatorname{Geo}\left(\frac{1}{2}\right)$, where $X=\operatorname{Ber}(\alpha)$ is a Bernoulli-distributed random variable independent of $\operatorname{Geo}\left(\frac{1}{2}\right)$. A similar result is given by Mahmoud for the distances-however, normalization is necessary in this case to make the random variables converge.

Date: November 17, 2007. 
Wilson [9], on the other hand, creates an algebraic framework around the problem, and derives all results from a trivariate "grand" generating function.

In this paper we are interested in fixing two elements $j<k$ and consider the joint distribution of moves. We believe that the joint distribution of the respective distances could also be treated along the lines of this paper, but it seems that this involves even lengthier and more complicated calculations. Our methods are also suitable (at least in principle) to deal with any fixed number of elements $j_{1}<\cdots<j_{p}$, but considering the remarkable complexity of the computations we confine ourselves to the instance $p=2$.

As Mark Wilson [10] points out, Sattolo's algorithm to create a random cyclic permutation is very similar to the Fisher-Yates algorithm to construct a random permution [1], and so he gives a unified approach to both of them. The difference to Sattolo's algorithm lies in the fact that the random integer chosen in the $i$-th step lies between 1 and $n+1-i$ rather than between 1 and $n-i$. If $n+1-i$ is chosen, it is interchanged with itself, and a cycle is closed. Our methods also apply to the analysis of this algorithm, so we will again study the joint distribution of the moves of two elements.

The outline of this paper is as follows: In Section $2, \phi_{n, k}^{(1)}(u)$, the probability generating function for the number of moves of a single element $k$ in applying Sattolo's algorithm for the generation of a random cyclic permutation of $n$ elements is reconsidered, to make the treatment self-contained. The recursion can be solved by elementary means in this case. Then we come to deal with the central theme of this paper, $\phi_{n, k, j}^{(2)}(u, v)$, the bivariate generating function for the number of moves of two elements $k$ and $j$. Somewhat surprisingly, it is still possible to solve the recursion for this function explicitly (Theorem 1), which is a remarkable tour de force. Indeed, we believe that the method of repeated summing and taking differences can also be applied in other circumstances.

As a corollary, we can deduce moments and determine the grand average, the average of $\phi_{n, k, j}^{(2)}(u, v)$ over all possible pairs $(k, j)$ with $k>j$. In Section 3 , the explicit formulæ are evaluated asymptotically. We let $k$ and $j$ grow proportionally to $n$ and find that, asymptotically, the numbers of moves become independent (dependencies in lower order terms are explicitly computed). Of course, the marginal distributions (of the moves of a single element) are as described in Mahmoud's paper.

Sections 4 and 5 are concerned with similar results for the Fisher-Yates algorithm. In a final section, we compute the probability that two elements $i, j$ change place during either Sattolo's or Fisher-Yates' algorithm. This is done by setting up appropriate recursions and solving them.

Let us make a brief statement about the motivation and the impact of the present research.

- In several questions about algorithms it is important to know what happens to two elements, when there are dependencies between the elements. As an example we would like to mention a paper that one of the authors wrote together with A. Panholzer [5], following on earlier ideas in [2] and [6]. Another example is [3], where it is shown how the variance can be computed, once the joint behaviour of two elements (rows in a matrix) is well-understood. This seems to be the way to go if more explicit quantities are not available.

- We also believe that the technique we use to handle the recursions in this paper is relevant on its own right and also applicable in other contexts when a generating function of two variables has to be analysed. It involves iterated summing and taking 
differences, and the fact that the variables change places results in higher-order recursions. The use of computer algebra is helpful, of course, but careful human guidance is essential. Finally, it is interesting and by no means automatic that such a recursion with polynomial coefficients has a reasonably "nice" solution, as was fortunately the case here.

We frequently use the notation of harmonic numbers:

$$
H_{n}=\sum_{1 \leq k \leq n} \frac{1}{k}, \quad H_{n}^{(2)}=\sum_{1 \leq k \leq n} \frac{1}{k^{2}}
$$

and Iverson's notation: $[P]=1$ if the condition $P$ is true, 0 otherwise.

\section{The number of moves in SATtolo's Algorithm}

First we repeat a few facts on $\phi_{n, k}^{(1)}(u)$, the probability generating function for the number of moves of a single element $k$ in applying Sattolo's algorithm to a set of $n$ elements. We use an upper index to distinguish this generating function from $\phi_{n, k, j}^{(2)}(u, v)$ which will denote the joint probability generating function of two elements $j<k$, where the variable $u$ refers to the moves of $k$, and $v$ to the moves of $j$.

The following recursions for $\phi^{(1)}$ are already known (see $[4,7,9]$ ):

$$
\begin{gathered}
\phi_{n, k}^{(1)}(u)=\frac{n-k}{n-1} u+\frac{k-1}{n-1} \phi_{k, k}^{(1)}(u), \quad 1 \leq k<n, \\
\phi_{n, n}^{(1)}(u)=\frac{u}{n-1} \sum_{k=1}^{n-1} \phi_{n-1, k}^{(1)}(u), \quad n \geq 2, \phi_{1,1}^{(1)}(u)=1 .
\end{gathered}
$$

Note that the first formula also holds for $k=n$, but it is quite meaningless in this case.

The strategy to solve such a recursion is to replace the terms in the second sum using the first recursion - the same approach will be also used for the joint generating function:

$$
\begin{aligned}
\phi_{n, n}^{(1)}(u) & =\frac{u}{n-1} \sum_{k=1}^{n-1}\left[\frac{n-1-k}{n-2} u+\frac{k-1}{n-2} \phi_{k, k}^{(1)}(u)\right] \\
& =\frac{u^{2}}{2}+\frac{u}{(n-1)(n-2)} \sum_{k=1}^{n-1}(k-1) \phi_{k, k}^{(1)}(u) .
\end{aligned}
$$

It is natural to multiply this by $(n-1)(n-2)$ :

$$
(n-1)(n-2) \phi_{n, n}^{(1)}(u)=\frac{(n-1)(n-2) u^{2}}{2}+u \sum_{k=1}^{n-1}(k-1) \phi_{k, k}^{(1)}(u) .
$$

Taking differences, we get

$$
(n-1) \phi_{n, n}^{(1)}(u)=u^{2}+(n-3+u) \phi_{n-1, n-1}^{(1)}(u)
$$

or, making use of the initial value $\phi_{2,2}^{(1)}(u)=u$,

$$
\frac{(n-1) !}{(n-3+u) !} \phi_{n, n}^{(1)}(u)=\frac{(n-2) ! u^{2}}{(n-3+u) !}+\frac{(n-2) !}{(n-4+u) !} \phi_{n-1, n-1}^{(1)}(u)
$$




$$
\begin{aligned}
& =u^{2} \sum_{k=3}^{n} \frac{(k-2) !}{(k-3+u) !}+\frac{u}{(u-1) !} \\
& =u^{2} \frac{(n-1) !}{(n-3+u) !(2-u)}-\frac{u^{2}}{(2-u)(u-1) !}+\frac{u}{(u-1) !},
\end{aligned}
$$

whence we find

$$
\phi_{n, n}^{(1)}(u)= \begin{cases}\frac{u^{2}}{2-u}-\frac{2 u}{2-u}\left(\begin{array}{c}
n+u-3 \\
n-1
\end{array}\right) & n>1 \\
1 & n=1\end{cases}
$$

and

$$
\begin{aligned}
\phi_{n, k}^{(1)}(u) & =\frac{n-k}{n-1} u+\frac{k-1}{n-1} \phi_{k, k}^{(1)}(u) \\
& = \begin{cases}\frac{u(n u-2 k u-2 n+2 k+u)}{(u-2)(n-1)}+\frac{2 u(k-1)}{(n-1)(u-2)}\left(\begin{array}{cl}
k+u-3 \\
k-1
\end{array}\right) & n \geq k>1, \\
u & n>k=1, \\
1 & n=k=1 .\end{cases}
\end{aligned}
$$

It is also interesting to compute the grand average generating function in this case; compare for instance $[2,5]$ :

$$
\frac{1}{n} \sum_{1 \leq k \leq n} \phi_{n, k}^{(1)}(u)=\frac{u}{2-u}-\frac{2}{2-u}\left(\begin{array}{c}
n+u-2 \\
n
\end{array}\right) .
$$

Remark. If we set

$$
G(z):=\sum_{n=2}^{\infty} z^{n-1} \phi_{n, n}^{(1)}(u)
$$

then the recursion just obtained translates to the differential equation

$$
z^{2} G^{\prime \prime}(z)=\frac{u^{2} z^{2}}{(1-z)^{3}}+\frac{u z}{1-z} G^{\prime}(z)-u z G^{\prime}(z), \quad G(0)=0, G^{\prime}(0)=u,
$$

with the solution

$$
G(z)=-\frac{2 u}{(2-u)(1-z)^{u-1}}+\frac{u^{2} z}{(2-u)(1-z)}+\frac{2 u}{2-u} .
$$

The last term induces $\left[z^{0}\right] G(z)=0$ as it should. Note that $G(z)$ was obtained by Wilson, $[9$, p. 304], in a complicated manner starting from a "grand" generating function.

The solution for $G(z)$ leads again to

$$
\phi_{n, n}^{(1)}(u)=\left[z^{n-1}\right] G(z)=\frac{u^{2}}{2-u}-\frac{2 u}{2-u}\left(\begin{array}{c}
n-3+u \\
n-1
\end{array}\right),
$$

which is also exactly the formula obtained by Mahmoud [4, p. 792] by means of a randomizationderandomization technique.

However, we will not go the route of differential equations in our treatment of two elements, since it becomes quite unmanageable.

It is possible to derive all moments from equation (1). For instance, using the relation $\psi(n)=-\gamma+H_{n-1}$ for the Digamma function, we obtain

$$
\mathbb{E}\left(X_{k}\right)=\frac{2 k+n-5}{n-1} \quad \text { and }
$$




$$
\mathbb{V}\left(X_{k}\right)=\frac{2(k-2)(3 n+1-2 k)}{(n-1)^{2}}-\frac{4 H_{k-2}}{n-1} .
$$

These have already been given by Prodinger in [7].

Now we consider, for $1 \leq j<k \leq n$, the generating function $\phi_{n, k, j}^{(2)}(u, v)$, where the coefficient of $u^{a} v^{b}$ is the probability that $k$ makes $a$ and $j$ makes $b$ moves. Using a similar approach as for $\phi^{(1)}$, that involves summing and taking differences in several steps, we are able to determine an explicit formula for this generating function. In this process, lengthy hypergeometric terms arise, which are best treated by a computer algebra system.

$\phi^{(2)}$ is determined by the following recursions, which are obtained in the same manner as the recursions for $\phi^{(1)}$ : for $1 \leq j<n$,

$$
\phi_{n, n, j}^{(2)}(u, v)=\frac{u}{n-1}\left[\sum_{l=1}^{j-1} \phi_{n-1, j, l}^{(2)}(v, u)+\sum_{l=j+1}^{n-1} \phi_{n-1, l, j}^{(2)}(u, v)+v \phi_{n-1, j}^{(1)}(u)\right],
$$

and for $1 \leq j<k<n$ (and also for $k=n$, but this is not of any particular use)

$$
\begin{aligned}
\phi_{n, k, j}^{(2)}(u, v) & =\frac{(k-1)(k-2)}{(n-1)(n-2)} \phi_{k, k, j}^{(2)}(u, v) \\
& +\sum_{l=k}^{n-1} \frac{l-1}{(n-1)(n-2)} u \phi_{l, j}^{(1)}(v)+\sum_{l=k}^{n-1} \frac{l-1}{(n-1)(n-2)} v \phi_{l, k}^{(1)}(u) .
\end{aligned}
$$

Indeed, the first term corresponds to the event " $k, j$ are not selected between step 1 and step $n-k$ " (whose probability is easily seen to be $\frac{(k-1)(k-2)}{(n-1)(n-2)}$ ), the first sum corresponds to the event " $k$ is selected within these steps (before $j$ )", and the second sum corresponds to the event " $j$ is selected within these steps (before $k$ )", with $l$ elements remaining in the latter two cases. The first equation can be justified by similar arguments. From the explicit formulæ for $\phi_{n, k}^{(1)}(u)$ derived above, we conclude that

$$
\begin{aligned}
& \sum_{l=k}^{n-1} \frac{l-1}{(n-1)(n-2)} u \phi_{l, j}^{(1)}(v) \\
& =\sum_{l=k}^{n-1} \frac{l-1}{(n-1)(n-2)} u\left[\frac{v(l v-2 j v-2 l+2 j+v)}{(v-2)(l-1)}+\frac{2 v(j-1)}{(l-1)(v-2)}\left(\begin{array}{c}
j+v-3 \\
j-1
\end{array}\right)\right] \\
& =\frac{u v}{(n-1)(n-2)(v-2)} \sum_{l=k}^{n-1}\left[l v-2 j v-2 l+2 j+v+2(j-1)\left(\begin{array}{c}
j+v-3 \\
j-1
\end{array}\right)\right] \\
& =\frac{u v(n-k)}{(n-1)(n-2)(v-2)}\left[\frac{(v-2)(n+k-1)}{2}-2 j v+2 j+v+2(j-1)\left(\begin{array}{c}
j+v-3 \\
j-1
\end{array}\right)\right]
\end{aligned}
$$

for $j>1$ and

$$
\sum_{l=k}^{n-1} \frac{l-1}{(n-1)(n-2)} u \phi_{l, 1}^{(1)}(v)=\frac{u v(n-k)(n+k-3)}{2(n-1)(n-2)} .
$$


Similarly,

$$
\begin{aligned}
& \sum_{l=k}^{n-1} \frac{n-l-1}{(n-1)(n-2)} v \phi_{n-l, k}^{(1)}(u) \\
& =\frac{u v(n-k)}{(n-1)(n-2)(u-2)}\left[\frac{(u-2)(n+k-1)}{2}-2 k u+2 k+u+2(k-1)\left(\begin{array}{c}
k+u-3 \\
k-1
\end{array}\right)\right] .
\end{aligned}
$$

Altogether, this yields a simplified version (not containing $\phi^{(1)}$ any more) of the recursions:

$$
\begin{aligned}
\phi_{n, k, j}^{(2)}(u, v) & =\frac{(k-1)(k-2)}{(n-1)(n-2)} \phi_{k, k, j}^{(2)}(u, v) \\
+ & \frac{u v(n-k)}{(n-1)(n-2)}\left[n+k-1-\frac{2 j v-2 j-v}{v-2}-\frac{2 k u-2 k-u}{u-2}\right. \\
& \left.+\frac{2(v-1)}{v-2}\left(\begin{array}{c}
j+v-3 \\
j-2
\end{array}\right)+\frac{2(u-1)}{u-2}\left(\begin{array}{c}
k+u-3 \\
k-2
\end{array}\right)\right]
\end{aligned}
$$

for $j>1$ and

$$
\begin{aligned}
\phi_{n, k, j}^{(2)}(u, v)=\frac{(k-1)(k-2)}{(n-1)(n-2)} \phi_{k, k, j}^{(2)}(u, v)+\frac{u v(n-k)}{(n-1)(n-2)(u-2)} \\
\times\left[(u-2)(n+k-2)-2 k u+2 k+u+2(k-1)\left(\begin{array}{c}
k+u-3 \\
k-1
\end{array}\right)\right]
\end{aligned}
$$

for $j=1$. Thus, for $1 \leq j<n$,

$$
\begin{aligned}
\phi_{n, n, j}^{(2)}(u, v) & =\frac{u}{n-1}\left[\sum_{l=1}^{j-1} \phi_{n-1, j, l}^{(2)}(v, u)+\sum_{l=j+1}^{n-1} \phi_{n-1, l, j}^{(2)}(u, v)+v \phi_{n-1, j}^{(1)}(u)\right] \\
& =\frac{u}{n-1}\left[\sum_{l=1}^{j-1} \frac{(j-1)(j-2)}{(n-2)(n-3)} \phi_{j, j, l}^{(2)}(v, u)+\sum_{l=j+1}^{n-1} \frac{(l-1)(l-2)}{(n-2)(n-3)} \phi_{l, l, j}^{(2)}(u, v)+X_{1}\right],
\end{aligned}
$$

where $X_{1}$ is given by

$$
\begin{aligned}
& X_{1}:=\frac{u v(n-j-1)}{(n-2)(n-3)(v-2)}\left[(v-2)(n+j-3)-2 j v+2 j+v+2(j-1)\left(\begin{array}{c}
j+v-3 \\
j-1
\end{array}\right)\right] \\
& +\sum_{l=2}^{j-1} \frac{u v(n-j-1)}{(n-2)(n-3)}\left[n+j-2-\frac{2 l u-2 l-u}{u-2}-\frac{2 j v-2 j-v}{v-2}\right. \\
& \left.+\frac{2(u-1)}{u-2}\left(\begin{array}{c}
l+u-3 \\
l-2
\end{array}\right)+\frac{2(v-1)}{v-2}\left(\begin{array}{c}
j+v-3 \\
j-2
\end{array}\right)\right] \\
& +\sum_{l=j+1}^{n-1} \frac{u v(n-l-1)}{(n-2)(n-3)}\left[n+l-2-\frac{2 j v-2 j-v}{v-2}-\frac{2 l u-2 l-u}{u-2}+\frac{2(v-1)}{v-2}\left(\begin{array}{c}
j+v-3 \\
j-2
\end{array}\right)\right. \\
& \left.+\frac{2(u-1)}{u-2}\left(\begin{array}{c}
l+u-3 \\
l-2
\end{array}\right)\right] \\
& +\frac{u v(n u-2 j u-2 n+2 j+2)}{(u-2)(n-2)}+\frac{2 u v(j-1)}{(n-2)(u-2)}\left(\begin{array}{c}
j+u-3 \\
j-1
\end{array}\right)
\end{aligned}
$$


for $j>1$ and by

$X_{1}:=\sum_{l=2}^{n-1} \frac{u v(n-l-1)}{(n-2)(n-3)(u-2)}\left[(u-2)(n+l-3)-2 l u+2 l+u+2(l-1)\left(\begin{array}{c}l+u-3 \\ l-1\end{array}\right)\right]+u v$

for $j=1$. The sums of hypergeometric terms can be computed explicitly. After some simplifications, we see that this is equivalent to

$$
\begin{aligned}
X_{1} & =\frac{u v}{3(n-2)(n-3)(u-2)(v-2)}\left[n^{3}(u-3)(v-2)-3 n^{2} j(v-1)(u-2)\right. \\
& -3 n^{2}(10-3 u-4 v+u v)+15 n j(v-1)(u-2)-n(4 u v+3 v+7 u-36)+j^{3}(2 u v-u-3 v) \\
& -3 j^{2}(4 u v-5 v-4 u+2)+j(4 u v+12 v-11 u-18) \\
& +6(2 u-v-2)+6(u-1)^{2}(v-2)\left(\begin{array}{c}
j+u-3 \\
j-3
\end{array}\right)-6(u-1)(v-2)\left(\begin{array}{c}
j+u-3 \\
j-4
\end{array}\right) \\
& \left.+6(u-1)(v-2)\left(\begin{array}{c}
n+u-3 \\
n-4
\end{array}\right)+3(n-j-1)(n+j-4)(u-2)(v-1)\left(\begin{array}{c}
j+v-3 \\
j-2
\end{array}\right)\right]
\end{aligned}
$$

for $j>1$ and to

$$
X_{1}=\frac{u v}{3(n-2)(u+1)(u-2)}\left[(n-2)(n-1)(u-3)(u+1)+6(u-1)\left(\begin{array}{c}
n+u-3 \\
n-3
\end{array}\right)\right]
$$

for $j=1$. However, this is exactly the value of the above expression if we take the limit $j \rightarrow 1$, so we don't have to distinguish between $j=1$ and $j>1$ any longer. Now we set $(n-1)(n-2) \phi_{n, n, j}^{(2)}(u, v)=R_{n, j}(u, v)$ to obtain

$$
(n-3) R_{n, j}(u, v)=u \sum_{l=1}^{j-1} R_{j, l}(v, u)+u \sum_{l=j+1}^{n-1} R_{l, j}(u, v)+X_{2}
$$

with $X_{2}:=u(n-2)(n-3) X_{1}$. Taking differences, it follows that

$$
\begin{aligned}
& (n-3) R_{n, j}(u, v)-(n-4) R_{n-1, j}(u, v) \\
& \quad=u \sum_{l=1}^{j-1} R_{j, l}(v, u)+u \sum_{l=j+1}^{n-1} R_{l, j}(u, v)-u \sum_{l=1}^{j-1} R_{j, l}(v, u)-u \sum_{l=j+1}^{n-2} R_{l, j}(u, v)+X_{3} \\
& \quad=u R_{n-1, j}(u, v)+X_{3}
\end{aligned}
$$

or

$$
\frac{(n-3) !}{(n-4+u) !} R_{n, j}(u, v)=\frac{(n-4) !}{(n-5+u) !} R_{n-1, j}(u, v)+\frac{(n-4) !}{(n-4+u) !} X_{3}
$$

with

$$
\begin{aligned}
X_{3}:=\frac{u^{2} v}{(u-2)(v-2)} & {[(n-3)((u-3)(v-2) n-2(u-2)(v-1) j+2(u+v-4))} \\
+ & \left.2(n-3)(u-2)(v-1)\left(\begin{array}{c}
j+v-3 \\
j-2
\end{array}\right)+2(u-1)(v-2)\left(\begin{array}{c}
n+u-4 \\
n-4
\end{array}\right)\right] .
\end{aligned}
$$


We set $S_{n, j}(u, v)=\frac{(n-3) !}{(n-4+u) !} R_{n, j}(u, v)=\frac{(n-1) !}{(n-4+u) !} \phi_{n, n, j}^{(2)}(u, v)$ and obtain

$$
\begin{aligned}
& S_{n, j}(u, v)= S_{n-1, j}(u, v) \\
&+ \frac{u^{2} v}{(u-2)(v-2)}\left[\frac{(n-3) !}{(n-4+u) !}[(u-3)(v-2) n-2(u-2)(v-1) j\right. \\
&\left.\left.\quad+2(u+v-4)+2(u-2)(v-1)\left(\begin{array}{c}
j+v-3 \\
j-2
\end{array}\right)\right]+\frac{2(u-1)(v-2)}{u !}\right] \\
&=S_{n-1, j}(u, v)+\frac{u^{2} v(u-3)}{u-2} \cdot \frac{(n-2) !}{(n-4+u) !} \\
& \quad+\frac{2 u^{2} v(v-1)}{v-2}\left[\left(\begin{array}{c}
j+v-3 \\
j-2
\end{array}\right)-j+1\right] \frac{(n-3) !}{(n-4+u) !}+\frac{2 u v}{(u-2) !(u-2)} .
\end{aligned}
$$

Summing this for $n=j+2, \ldots, N$ yields

$$
\begin{aligned}
S_{N, j}(u, v)=S_{j+1, j}(u, v)+\frac{u^{2} v}{u-2}\left[\frac{j !}{(j+u-3) !}-\frac{(N-1) !}{(N+u-4) !}\right] \\
+\frac{2 u^{2} v(v-1)}{(u-2)(v-2)}\left[\left(\begin{array}{c}
j+v-3 \\
j-2
\end{array}\right)-j+1\right] \cdot\left[\frac{(j-1) !}{(j+u-3) !}-\frac{(N-2) !}{(N+u-4) !}\right] \\
+\frac{2 u v(N-j-1)}{(u-2) !(u-2)} .
\end{aligned}
$$

It remains to compute $S_{j+1, j}(u, v)$, that is, we need $R_{j+1, j}(u, v)$. For this purpose, we take $n=j+1$ in the recursive formula for $R_{n, j}(u, v)$ :

$$
(j-2) R_{j+1, j}(u, v)=u \sum_{l=1}^{j-1} R_{j, l}(v, u)+\frac{u^{3} v}{u-2}\left[2(u-1)\left(\begin{array}{c}
j+u-3 \\
j-3
\end{array}\right)-(j-1)(j-2)\right],
$$

which can be rewritten as

$$
\begin{aligned}
\frac{(j-2)(j-3+u) !}{(j-2) !} S_{j+1, j}(u, v) & =u \sum_{l=1}^{j-1} \frac{(j-4+v) !}{(j-3) !} S_{j, l}(v, u) \\
& +\frac{u^{3} v}{u-2}\left[2(u-1)\left(\begin{array}{c}
j+u-3 \\
j-3
\end{array}\right)-(j-1)(j-2)\right],
\end{aligned}
$$

which in turn reduces to

$$
S_{j+1, j}(u, v)=\frac{u(j-4+v) !}{(j-3+u) !} \sum_{l=1}^{j-1} S_{j, l}(v, u)+\frac{u^{3} v}{u-2}\left[\frac{2(u-1)}{u !}-\frac{(j-1) !}{(j-3+u) !}\right] .
$$

Now we express $S_{j, l}(v, u)$ in terms of $S_{l+1, l}(v, u)$ :

$$
\begin{aligned}
S_{j, l}(v, u)=S_{l+1, l}(v, u)+\frac{v^{2} u}{v-2} \cdot\left[\frac{l !}{(l+v-3) !}-\frac{(j-1) !}{(j+v-4) !}\right] \\
+\frac{2 v^{2} u(u-1)}{(v-2)(u-2)}\left[\left(\begin{array}{c}
l+u-3 \\
l-2
\end{array}\right)-l+1\right] \cdot\left[\frac{(l-1) !}{(l+v-3) !}-\frac{(j-2) !}{(j+v-4) !}\right] \\
+\frac{2 v u(j-l-1)}{(v-2) !(v-2)}
\end{aligned}
$$




$$
\begin{aligned}
&=S_{l+1, l}(v, u)+\frac{2 u v(j-1)}{(v-2)(v-2) !}-\frac{u v^{2}(j u+u-2 j)(j-2) !}{(u-2)(v-2)(j+v-4) !} \\
&+\frac{2 u v l}{v-2}\left[\frac{v(u-1)(j-2) !}{(u-2)(j+v-4) !}-\frac{1}{(v-2) !}\right] \\
&+\frac{2 v^{2} u(u-1)}{(u-2)(v-2)} \cdot \frac{(l-1) !}{(l+v-3) !}-\frac{v^{2} u^{2}}{(u-2)(v-2)} \cdot \frac{l !}{(l+v-3) !} \\
&-\frac{2 u v^{2}(u-1)(j-2) !}{(u-2)(v-2)(j+v-4) !}\left(\begin{array}{c}
l+u-3 \\
l-2
\end{array}\right) \\
&+\frac{2 u v^{2}}{(u-2)(v-2)(u-2) !} \cdot \frac{(l+u-2) !}{(l+v-3) !}-\frac{2 u v^{2}(u-1)}{(u-2)(v-2)(u-2) !} \cdot \frac{(l+u-3) !}{(l+v-3) !} .
\end{aligned}
$$

Summing this for $l=1, \ldots, j-1$ yields

$$
\begin{aligned}
\sum_{l=1}^{j-1} S_{j, l}(v, u)=\sum_{l=1}^{j-1} & S_{l+1, l}(v, u)+\frac{2 u v(j-1)^{2}}{(v-2)(v-2) !}-\frac{u v^{2}(j u+u-2 j)(j-1) !}{(u-2)(v-2)(j+v-4) !} \\
& +\frac{u v j(j-1)}{v-2}\left[\frac{v(u-1)(j-2) !}{(u-2)(j+v-4) !}-\frac{1}{(v-2) !}\right] \\
& -\frac{2 v^{2} u(u-1)}{(u-2)(v-2)(v-3)}\left[\frac{(j-1) !}{(j+v-4) !}-\frac{1}{(v-3) !}\right] \\
& +\frac{v^{2} u^{2}}{(u-2)(v-2)(v-4)}\left[\frac{j !}{(j+v-4) !}-\frac{1}{(v-3) !}\right] \\
& -\frac{2 v^{2}(j-2) !}{(u-2)(v-2)(j+v-4) !(u-2) !} \cdot \frac{(j+u-3) !}{(j-3) !} \\
& +\frac{2 u v^{2}}{(u-2)(v-2)(u-2) !(u-v+2)}\left[\frac{(j+u-2) !}{(j+v-4) !}-\frac{(u-1) !}{(v-3) !}\right] \\
& -\frac{2 u v^{2}(u-1)}{(u-2)(v-2)(u-2) !(u-v+1)}\left[\frac{(j+u-3) !}{(j+v-4) !}-\frac{(u-2) !}{(v-3) !}\right] .
\end{aligned}
$$

Now, it follows that

$$
\frac{(j-3+u) !}{u(j-4+v) !} S_{j+1, j}(u, v)=\sum_{l=1}^{j-1} S_{l+1, l}(v, u)+Q_{j}(u, v)
$$

where

$$
\begin{aligned}
Q_{j}(u, v)= & \frac{u v(j-1)(j-2)}{(v-2)(v-2) !}+\frac{u v^{2}\left(v^{3}-2 u v^{2}+u^{2} v-6 v^{2}+13 u v-5 u^{2}+7 v-17 u+4\right)}{(u-v+1)(u-v+2)(v-4)(v-3)(v-2) !} \\
& -\frac{2 u v\left(u v^{2}-3 u v-v+3 u\right)(j-1) !}{(u-2)(v-3)(v-2)(j-4+v) !}+\frac{u v^{2}(u+v-4) j !}{(u-2)(v-2)(v-4)(j-4+v) !} \\
& +\frac{2 u v(u-2 v+1)(j-3+u) !}{(u-2)(u-v+1)(u-2) !(j-4+v) !}+\frac{2 v^{2}(j-2+u) !}{(u-2)(u-v+2)(u-2) !(j-4+v) !},
\end{aligned}
$$


which means that the problem has been reduced to a recursion for the one-dimensional sequence $S_{j+1, j}(u, v)$. Elimination yields

$\frac{(j-2+u) !}{u(j-3+v) !} S_{j+2, j+1}(u, v)-\frac{(j-3+u) !}{u(j-4+v) !} S_{j+1, j}(u, v)=S_{j+1, j}(v, u)+Q_{j+1}(u, v)-Q_{j}(u, v)$, and we have

$$
\begin{aligned}
P_{j}(u, v):= & Q_{j+1}(u, v)-Q_{j}(u, v) \\
= & \frac{2 u v(j-1)}{(v-2)(v-2) !}+\frac{2 u v\left(u v^{2}-3 u v-v+3 u\right)(j-1) !}{(u-2)(v-2)(j-3+v) !} \\
& \quad-\frac{u v^{2}(u+v-4) j !}{(u-2)(v-2)(j-3+v) !}+\frac{2 u v(u-2 v+1)(j-3+u) !}{(u-2)(u-2) !(j-3+v) !} \\
& \quad+\frac{2 v^{2}(j-2+u) !}{(u-2)(u-2) !(j-3+v) !} .
\end{aligned}
$$

Writing $C_{j}(u, v)=\frac{(j-3+u) !}{u(j-4+v) !}$, we obtain

$$
C_{j+1}(u, v) S_{j+2, j+1}(u, v)-C_{j}(u, v) S_{j+1, j}(u, v)=S_{j+1, j}(v, u)+P_{j}(u, v) .
$$

Interchanging $u$ and $v$ and combining the two equations, we arrive at

$$
\begin{aligned}
& C_{j+1}(v, u) C_{j+2}(u, v) S_{j+3, j+2}(u, v)-C_{j+1}(u, v)\left(C_{j}(v, u)+C_{j+1}(v, u)\right) S_{j+2, j+1}(u, v) \\
& +\left(C_{j}(u, v) C_{j}(v, u)-1\right) S_{j+1, j}(u, v)=C_{j+1}(v, u) P_{j+1}(u, v)-C_{j}(v, u) P_{j}(u, v)+P_{j}(v, u) .
\end{aligned}
$$

After some simplifications, we see that this is equivalent to

$$
\begin{aligned}
(j+u-1)(j+u-2) S_{j+3, j+2}(u, v)-(j & +u-2)(2 j+u+v-5) S_{j+2, j+1}(u, v) \\
& +(j-3)(j+u+v-3) S_{j+1, j}(u, v)=L_{j}(u, v),
\end{aligned}
$$

for $j \geq 2$, where $L_{j}(u, v)$ is given by

$$
\begin{aligned}
L_{j}(u, v)= & \frac{2 u v\left(j v(u+2)+u^{2}-u v-4 v+u\right)}{(u-2)(u-2) !} \\
& -\frac{2 u^{2} v(j-1) !\left(2 j v(u+v-4)-3(v-1)\left(u^{2}-3 u+v\right)\right)}{(u-2)(v-2)(j-3+u) !} \\
& +\frac{2 u^{2} v(j-3+v) !\left(j(v+2)+v^{2}-u v+v-u-3\right)}{(v-2)(v-2) !(j-3+u) !} .
\end{aligned}
$$

Setting $S_{j+1, j}(u, v)=s_{j-2}$ for $j \geq 2$, we obtain the recurrence

$$
(j+u+1)(j+u) s_{j+2}-(j+u)(2 j+u+v-1) s_{j+1}+(j-1)(j+u+v-1) s_{j}=h_{j}
$$

with

$$
\begin{aligned}
h_{j}= & \frac{2 u v(v(u+2) j+u(u+v+1))}{(u-2)(u-2) !}+\frac{6 u^{2}(v-1) v\left(u^{2}+v-3 u\right)(j+1) !}{(u-2)(v-2)(j+u-1) !} \\
- & \frac{4 u^{2} v^{2}(u+v-4)(j+2) !}{(u-2)(v-2)(j+u-1) !}+\frac{2 u^{2} v(2+v)(j+v) !}{(v-2)(u+j-1) !(v-2) !} \\
& -\frac{2 u^{2} v(u-1)(v+1)(j+v-1) !}{(v-2)(j+u-1) !(v-2) !}
\end{aligned}
$$


for $j \geq 0$. The initial values are given by $s_{0}=\frac{2 u^{2} v}{(u-1) !}$ and $s_{1}=\frac{2 u^{2} v(u+2 v)}{u !}$. The explicit solution to this recurrence is found to be

$$
\begin{aligned}
s_{j}=- & \frac{2 v u(v j+2 u)}{(v-2)(u-2)(u-2) !}-\frac{2 u^{2} v(j-u+v+2)(j+v-1) !}{(v-2)(u-2)(v-2) !(j+u-1) !} \\
& +\frac{u^{2} v(j+1) !\left(v(u+v-3) j-2\left(u^{2} v-u^{2}-4 u v+3 u-4 v\right)\right.}{(v-2)(u-2)(u+v-3)(j+u-1) !} \\
& +\frac{2 u^{2} v^{2}\left(u^{2}+u v+v^{2}-4 u-4 v+5\right)(j+u+v-2) !}{\left(u^{2} v+u v^{2}-2 u^{2}-7 u v-2 v^{2}+10 u+10 v-12\right)(u+v-2) !(j+u-1) !} .
\end{aligned}
$$

This was obtained by means of computer algebra, but it can be readily checked that it satisfies the recursion and the initial conditions. It follows that

$$
\begin{aligned}
S_{j+1, j}(u, v)=- & \frac{2 v u(v j+2 u-2 v)}{(v-2)(u-2)(u-2) !}-\frac{2 u^{2} v(j-u+v)(j+v-3) !}{(v-2)(u-2)(v-2) !(j+u-3) !} \\
& +\frac{u^{2} v(j-1) !\left(v(u+v-3) j-2\left(u^{2} v-u^{2}+v^{2}-3 u v+3 u-7 v\right)\right.}{(v-2)(u-2)(u+v-3)(j+u-3) !} \\
& +\frac{2 u^{2} v^{2}\left(u^{2}+u v+v^{2}-4 u-4 v+5\right)(j+u+v-4) !}{\left(u^{2} v+u v^{2}-2 u^{2}-7 u v-2 v^{2}+10 u+10 v-12\right)(u+v-2) !(j+u-3) !}
\end{aligned}
$$

and

$$
\begin{aligned}
S_{n, j}(u, v)= & \frac{2 u v((v-2) n-2(v-1) j+v-2 u+2)}{(u-2)(v-2)(u-2) !} \\
& -\frac{2 u^{2} v(u-1)(u-3)(v-1)(j-1) !}{(u-2)(v-2)(u+v-3)(j+u-3) !} \\
& +\frac{2 u^{2} v(u-v-1)(j+v-3) !}{(u-2)(v-2)(v-2) !(j+u-3) !} \\
& -\frac{u^{2} v(n-2) !}{(u-2)(v-2)(n+u-4) !}\left[2(v-1)\left(\begin{array}{c}
j+v-3 \\
j-2
\end{array}\right)+(v-2) n-2(v-1) j+v\right] \\
& +\frac{2 u^{2} v^{2}\left(u^{2}+u v+v^{2}-4 u-4 v+5\right)(j+u+v-4) !}{\left(u^{2} v+u v^{2}-2 u^{2}-7 u v-2 v^{2}+10 u+10 v-12\right)(u+v-2) !(j+u-3) !} .
\end{aligned}
$$

Now we obtain an explicit expression for $\phi_{n, n, j}^{(2)}(u, v)=\frac{(n-4+u) !}{(n-1) !} S_{n, j}(u, v)$ :

$$
\begin{aligned}
& \phi_{n, n, j}^{(2)}(u, v)=\frac{(n-4+u) !}{(n-1) !}\left[\frac{2 u v((v-2) n-2(v-1) j+v-2 u+2)}{(u-2)(v-2)(u-2) !}\right. \\
&-\frac{2 u^{2} v(u-1)(u-3)(v-1)(j-1) !}{(u-2)(v-2)(u+v-3)(j+u-3) !} \\
&+\frac{2 u^{2} v(u-v-1)(j+v-3) !}{(u-2)(v-2)(v-2) !(j+u-3) !} \\
&\left.+\frac{2 u^{2} v^{2}\left(u^{2}+u v+v^{2}-4 u-4 v+5\right)(j+u+v-4) !}{\left(u^{2} v+u v^{2}-2 u^{2}-7 u v-2 v^{2}+10 u+10 v-12\right)(u+v-2) !(j+u-3) !}\right] \\
&-\frac{u^{2} v}{(u-2)(v-2)(n-1)}\left[2(v-1)\left(\begin{array}{c}
j+v-3 \\
j-2
\end{array}\right)+(v-2) n-2(v-1) j+v\right] .
\end{aligned}
$$


Note that the expected expressions arise if we set $u=1$ or $v=1$. Finally, we get the following explicit formula for $\phi_{n, k, j}^{(2)}(u, v)$ in the case $j>1$ :

Theorem 2.1. For $1<j<k \leq n$, we have

$$
\begin{aligned}
& \phi_{n, k, j}^{(2)}(u, v)=\frac{(k-4+u) !}{(k-3) !(n-1)(n-2)}\left[\frac{2 u v((v-2) k-2(v-1) j+v-2 u+2)}{(u-2)(v-2)(u-2) !}\right. \\
&-\frac{2 u^{2} v(u-1)(u-3)(v-1)(j-1) !}{(u-2)(v-2)(u+v-3)(j+u-3) !} \\
&+\frac{2 u^{2} v(u-v-1)(j+v-3) !}{(u-2)(v-2)(v-2) !(j+u-3) !} \\
&\left.+\frac{2 u^{2} v^{2}\left(u^{2}+u v+v^{2}-4 u-4 v+5\right)(j+u+v-4) !}{\left(u^{2} v+u v^{2}-2 u^{2}-7 u v-2 v^{2}+10 u+10 v-12\right)(u+v-2) !(j+u-3) !}\right] \\
&+\frac{u v}{(u-2)(v-2)(n-1)(n-2)}\left[n^{2}(u-2)(v-2)-2 k n(u-1)(v-2)\right. \\
&-2 j n(u-2)(v-1)+(u v-4) n+4 j k(u-1)(v-1)-4 k(u-1)-4 j u(v-1)+2 u v] \\
&+\frac{2 u v(u-1)(n-k)}{(u-2)(n-1)(n-2)}\left(\begin{array}{c}
k+u-3 \\
k-2
\end{array}\right) \\
&+\frac{2 u v(v-1)(n u-2 k u+2 k-2 n+2 u)}{(u-2)(v-2)(n-1)(n-2)}\left(\begin{array}{c}
j+v-3 \\
j-2
\end{array}\right) .
\end{aligned}
$$

Again, the correct formulæ arise if we set $u=1$ or $v=1$. However, the case $j=1$ has to be treated separately - here, we have

$$
S_{n, 1}(u, v)=\frac{2 u v(n+u-3)}{(u-2)(u-2) !}-\frac{u^{2} v(n-1) !}{(u-2)(n+u-4) !}
$$

and thus

$$
\phi_{n, n, 1}^{(2)}(u, v)=\frac{2 u v(n+u-3) !}{(u-2)(u-2) !(n-1) !}-\frac{u^{2} v}{u-2} .
$$

Finally, it follows that

$$
\phi_{n, k, 1}^{(2)}(u, v)=\frac{u v(n u-2 k u+2 k-2 n+u)}{(u-2)(n-1)}+\frac{2 u v(k-1)}{(u-2)(n-1)}\left(\begin{array}{c}
k+u-3 \\
k-1
\end{array}\right) .
$$

Of course, this is a trivial result, since we must have $\phi_{n, k, 1}^{(2)}(u, v)=v \phi_{n, k}^{(1)}(u)$ in view of the fact that $\phi_{n, 1}^{(1)}(v)=v$ corresponds to a one-point-distribution. Note also that the special case $j=1$ can be obtained from the general formula by plugging in $j=2$. This can also be seen directly from the algorithm.

Once one has determined such an explicit formula, it is easy to draw several corollaries from it. For instance, if one is interested in the covariance, the quantity $\left.\frac{\partial^{2}}{\partial u \partial v} \phi^{(2)}(u, v)_{n, k, j}\right|_{u=v=1}$ has to be computed:

$$
\left.\frac{\partial^{2}}{\partial u \partial v} \phi_{n, k, j}^{(2)}(u, v)\right|_{u=v=1}=\frac{n^{2}+(2 k+2 j-11) n+4 H_{j-2}+4 j k-12 k-20 j+50}{(n-1)(n-2)} .
$$


Furthermore, if we set $u=v$, we obtain the generating function for the combined number of moves of $j$ and $k$. A computer algebra system readily computes mean

$$
\text { mean }=\frac{2(n+j+k-5)}{(n-1)}
$$

and variance:

$$
\begin{aligned}
\text { variance } & =\frac{2 n^{2}+\left(12 k+12 j-4 H_{k-2}-4 H_{j-2}-54\right) n+8 H_{k-2}+16 H_{j-2}+8 k j-40 k-56 j+164}{(n-1)(n-2)} \\
& + \text { mean }- \text { mean }^{2} .
\end{aligned}
$$

(For $j=1$, as always, one has to use $j=2$ in the formula.)

As in the univariate case, it is interesting (and easily doable) to determine the grand average generating function:

$$
\begin{aligned}
& G_{n}(u, v)=\frac{1}{\left(\begin{array}{c}
n \\
2
\end{array}\right)} \sum_{1 \leq j<k \leq n} \phi_{n, k, j}^{(2)}(u, v) \\
& =-\frac{4 u\left(u v^{2}+n u v-6+2 n+2 u\right)(n+v-2) !}{(n-1) n !(u-2)(v-2)(v+2)(v+1)(v-2) !} \\
& +\frac{4 v\left(-u^{3}+u^{2} v-n u^{2}-2 u^{2}+n u v+u-3 n u-4+2 v\right)(n+u-2) !}{(n-1) n !(u+2)(u+1)(u-2)(u-2) !(v-2)} \\
& -\frac{u v\left(-6 n v+n v^{2}+9 n-n u^{2}-6 u v+u^{2}+2 u v^{2}-v^{2}+8 v+10 u-2 u^{2} v-15\right)}{3(n-1)(u-2)(v-2)(u+v-3)} \\
& +\frac{4 u^{2} v(u+v-1)\left(u^{2}+u v+v^{2}-4 u-4 v+5\right)(n+u+v-3) !}{(n-1) n !(u-2)(v-2)(u+v-3)(u+v) !} .
\end{aligned}
$$

The following quantities can be computed immediately (and the grand averages of mean, variance and covariance follow):

$$
\begin{aligned}
\left.\frac{\partial}{\partial u} G_{n}(u, v)\right|_{u=1, v=1} & =\frac{7 n-11}{3(n-1)}, \\
\left.\frac{\partial}{\partial v} G_{n}(u, v)\right|_{u=1, v=1} & =\frac{5 n^{2}-13 n+12}{3(n-1) n}, \\
\left.\frac{\partial^{2}}{\partial u^{2}} G_{n}(u, v)\right|_{u=1, v=1} & =\frac{2\left(8 n^{2}-13 n+12-6 n H_{n}\right)}{3(n-1) n}, \\
\left.\frac{\partial^{2}}{\partial v^{2}} G_{n}(u, v)\right|_{u=1, v=1} & =\frac{2\left(4 n^{3}-11 n^{2}+12 n-12-6 n(n-2) H_{n}\right)}{3(n-1) n^{2}}, \\
\left.\frac{\partial^{2}}{\partial u \partial v} G_{n}(u, v)\right|_{u=1, v=1} & =\frac{2\left(2 n^{3}-7 n^{2}+5 n-2+2 n H_{n}\right)}{(n-1) n^{2}} .
\end{aligned}
$$

\section{Asymptotics for the moves in Sattolo's Algorithm}

In this section, we analyze the asymptotics of the joint distribution of the random variables $X_{k}$ and $X_{j}$, the number of moves of $k$ and $j$, respectively. Here, we take $k=\alpha n, j=\beta n$ with $0<\beta<\alpha<1$ and let $n, k, j \rightarrow \infty$. Let us rewrite the formula for first: $\phi_{n, k, j}^{(2)}$ :

$\phi_{n, k, j}^{(2)}(u, v)=F_{1}+F_{2}+F_{3}$, where 


$$
\begin{aligned}
F_{1}= & \frac{\Gamma(k-3+u)}{\Gamma(k-2)(n-1)(n-2)}\left(\frac{2 u v((v-2) k-2(v-1) j+v-2 u+2)}{(u-2)(v-2) \Gamma(u-1)}\right. \\
& -\frac{2 u^{2} v(u-1)(u-3)(v-1) \Gamma(j)}{(u-2)(v-2)(u+v-3) \Gamma(j+u-2)} \\
& +\frac{2 u^{2} v(u-v-1) \Gamma(j+v-2)}{(u-2)(v-2) \Gamma(v-1) \Gamma(j+u-2)} \\
& \left.+\frac{2 u^{2} v^{2}\left(u^{2}+u v+v^{2}-4 u-4 v+5\right) \Gamma(j+u+v-3)}{\left(u^{2} v+u v^{2}-2 u^{2}-7 u v-2 v^{2}+10 u+10 v-12\right) \Gamma(u+v-1) \Gamma(j+u-2)}\right), \\
F_{2}:= & \frac{u v}{(u-2)(v-2)(n-1)(n-2)}\left(n^{2}(u-2)(v-2)-2 k n(u-1)(v-2)-2 j n(u-2)(v-1)\right. \\
& +(u v-4) n+4 j k(u-1)(v-1)-4 k(u-1)-4 j u(v-1)+2 u v) \text { and } \\
F_{3}:= & \frac{2 u v(u-1)(n-k) \Gamma(k+u-2)}{(u-2)(n-1)(n-2) \Gamma(u) \Gamma(k-1)}+\frac{2 u v(v-1)(n u-2 k u+2 k-2 n+2 u) \Gamma(j+v-2)}{(u-2)(v-2)(n-1)(n-2) \Gamma(v) \Gamma(j-1)} .
\end{aligned}
$$

By Stirling's approximation we get

$$
\frac{\Gamma(n+a+1)}{\Gamma(n+1)} \sim n^{a}(1+\mathcal{O}(1 / n)), \quad n \rightarrow \infty,
$$

where $\mathcal{O}(\cdot)$ here and in the sequel depends on $a$.

Now, plugging in $k=\alpha n$ and $j=\beta n$ leads to

$$
\begin{aligned}
& F_{1} \sim \mathcal{O}\left(n^{u-2}\right)+\mathcal{O}\left(n^{-1}\right)+\mathcal{O}\left(n^{-3}\right)+\mathcal{O}\left(n^{u+v-4}\right), \\
& F_{2} \sim \frac{u v[(u-2)(v-2)-2 \alpha(u-1)(v-2)-2 \beta(u-2)(v-1)+4 \beta \alpha(u-1)(v-1)]}{(u-2)(v-2)}, \\
& F_{3} \sim \mathcal{O}\left(n^{u-2}\right)+\mathcal{O}\left(n^{v-2}\right) .
\end{aligned}
$$

Hence the limiting joint probability generating function is given by

$$
\psi_{\alpha, \beta}^{2}(u, v)=\frac{u v[(u-2)(v-2)-2 \alpha(u-1)(v-2)-2 \beta(u-2)(v-1)+4 \beta \alpha(u-1)(v-1)]}{(u-2)(v-2)} .
$$

Note that

$$
\begin{aligned}
& \psi_{\alpha, \beta}^{2}(1,1)=1, \\
& \psi_{\alpha, \beta}^{2}(u, 1)=\frac{u(-u+2+2 \alpha u-2 \alpha)}{2-u}=(1-\alpha) u+\alpha \frac{u^{2}}{2-u},
\end{aligned}
$$

which agrees with $[4$, p. 793]. Now note that we have the factorization

$$
\psi_{\alpha, \beta}^{2}(u, v)=\left((1-\alpha) u+\alpha \frac{u^{2}}{2-u}\right)\left((1-\beta) v+\beta \frac{v^{2}}{2-v}\right),
$$

which shows that the random variables $X_{k}$ and $X_{j}$ are asymptotically independent, with distribution $1+X \cdot \operatorname{Geo}\left(\frac{1}{2}\right)$, where $X$ is $\operatorname{Ber}(\alpha)(\operatorname{Ber}(\beta)$, respectively), as mentioned in the introduction [4, Theorem 1].

It is possible to refine these asymptotics, taking the $1 / n$ and $\ln n / n$ terms into account. We first have a contribution from the first term of $F_{1}$, which gives

$$
T_{1}:=-\frac{n^{u}}{n^{2}} \frac{2 \alpha^{u-1} u v(-\alpha v+2 \alpha+2 \beta v-2 \beta)}{(u-2)(v-2) \Gamma(u-1)} .
$$


The second term of $F_{1}$ leads to

$$
T_{2}:=-\frac{2 \alpha^{u-1} \beta^{2-u} u^{2} v(u-1)(u-3)(v-1)}{n(u-2)(v-2)(u+v-3)} .
$$

The third term of $F_{1}$ can be neglected; the fourth term gives

$$
T_{4}:=\frac{n^{u+v}}{n^{4}} \frac{2 \alpha^{u-1} \beta^{v-1} u^{2} v^{2}\left(u^{2}+u v+v^{2}-4 u-4 v+5\right)}{\left(u^{2} v+u v^{2}-2 u^{2}-7 u v-2 v^{2}+10 u+10 v-12\right) \Gamma(u+v-1)} .
$$

Now $F_{2}$ can be decomposed as $F_{2,0}+F_{2,1}$, where

$$
\begin{aligned}
F_{2,0}= & \psi_{\alpha, \beta}^{2}(u, v), \\
F_{2,1}:= & \frac{1}{n} \frac{2 u v N}{(u-2)(v-2)}, \quad \text { with } \\
N:= & 2 u v+4+4 \alpha u-4 \alpha-5 \beta u v+5 \beta u-3 u-3 v \\
& \quad-3 \alpha u v+3 \alpha v+6 \beta v-6 \beta+6 \beta \alpha u v-6 \alpha \beta u-6 \alpha \beta v+6 \alpha \beta .
\end{aligned}
$$

Finally, $F_{3}$ gives two summands $F_{3,1}+F_{3,2}$, with

$$
\begin{aligned}
& F_{3,1}:=-\frac{n^{u}}{n^{2}} \frac{2 \alpha^{u-1} u v(\alpha-1)}{(u-2) \Gamma(u-1)}, \\
& F_{3,2}:=-\frac{n^{v}}{n^{2}} \frac{2 \beta^{v-1} u v(-u+2 \alpha u-2 \alpha+2)}{(v-2)(u-2) \Gamma(v-1)} .
\end{aligned}
$$

We have

Theorem 3.1. $\phi_{n, k, j}^{(2)}(u, v) \sim F(u, v)$, with $F(u, v):=T_{1}+T_{2}+T_{4}+F_{2,0}+F_{2,1}+F_{3,1}+F_{3,2}$, given by equations (4)-(10).

Now, setting $v=1$, we obtain

$$
F(u, 1)=\frac{u(-u+2+2 \alpha u-2 \alpha)}{2-u}-\frac{1}{n} \frac{2(\alpha-1) u(u-1)}{u-2}-\frac{n^{u}}{n^{2}} \frac{2 u \alpha^{u-1}}{(2-u) \Gamma(u-1)},
$$

from which all asymptotic moments of $X_{k}$ (up to the terms of order $1 / n$ and $\ln n / n$ ) can be derived automatically. For instance, we obtain

$$
\begin{aligned}
& \mathbb{E}\left(X_{k}\right) \sim 1+2 \alpha+\frac{1}{n}(2 \alpha-4), \\
& \mathbb{E}\left(X_{k}^{2}\right) \sim 1+10 \alpha+\frac{1}{n}(-20+10 \alpha-4 \gamma-4 \ln \alpha)-4 \frac{\ln n}{n}, \\
& \mathbb{V}\left(X_{k}\right) \sim 6 \alpha-4 \alpha^{2}-4 \frac{\ln n}{n}+\frac{1}{n}\left(22 \alpha-12-4 \gamma-4 \ln \alpha-8 \alpha^{2}\right) .
\end{aligned}
$$

Of course, these could also have been derived from (2) and (3).

Next, all cross-moments can be computed. We only give the asymptotic covariance here:

$$
\mathbb{E}\left(X_{k} X_{j}\right)=\left.\frac{\partial^{2} F(u, v)}{\partial u \partial v}\right|_{u=1, v=1} \sim 4 \alpha \beta+1+2 \beta+2 \alpha+\frac{1}{n}(12 \alpha \beta-8-14 \beta-6 \alpha)+\mathcal{O}\left(\frac{\ln n}{n^{2}}\right),
$$

$\operatorname{Cov}\left(X_{k}, X_{j}\right)=\left.\frac{\partial^{2} F(u, v)}{\partial u \partial v}\right|_{u=1, v=1}-\mathbb{E}\left(X_{k}\right) \mathbb{E}\left(X_{j}\right) \sim \frac{1}{n}(-8 \beta+4 \alpha \beta)+\mathcal{O}\left(\frac{\ln n}{n^{2}}\right)$.

Now we move to the asymptotics of the grand average generating function. We denote by $Y_{1}$ 
and $Y_{2}$ the random variables related to $u$ and $v$ respectively. Proceeding as before, we obtain a limiting joint distribution function for the grand average:

Theorem 3.2. The grand average function $G_{n}(u, v)=\frac{1}{\left(\begin{array}{c}n \\ 2\end{array}\right)} \sum_{1 \leq j<k \leq n} \phi_{n, k, j}^{(2)}(u, v)$ is asymptotically equal to $G(u, v)$, where

$$
\begin{aligned}
G(u, v) & :=G_{1}+G_{2}+G_{3}+G_{4}, \\
G_{1} & :=-\frac{4 n^{v} u(u v+2)}{(u-2)(v+1) \Gamma(v-1)\left(v^{2}-4\right) n^{2}}, \\
G_{2} & :=\frac{4 n^{u} v\left(-u^{2}+u v-3 u\right)}{(u+1) \Gamma(u-1)(v-2)\left(u^{2}-4\right) n^{2}}, \\
G_{3} & :=-\frac{u v(-u+v-3)}{3(u-2)(v-2)}-\frac{2 u v\left(-3 u v+u v^{2}+v+5 u-u^{2} v-3\right)}{3(u-2)(v-2)(u+v-3) n}, \\
G_{4} & :=\frac{4 n^{u+v}\left(u^{2}+u v+v^{2}-4 u-4 v+5\right)(u+v-1) u^{2} v}{(u-2)(v-2)(u+v-3) \Gamma(u+v+1) n^{4}} .
\end{aligned}
$$

Setting $v=1$, we obtain

$$
G(u, 1)=-\frac{u(u+2)}{3(u-2)}-\frac{2 u(u-1)}{3(u-2) n}+\frac{4 u n^{u}}{\left(u^{2}-u-2\right) \Gamma(u-1) n^{2}},
$$

from which we derive the moments

$$
\begin{aligned}
& \mathbb{E}\left(Y_{1}\right) \sim \frac{7}{3}-\frac{4}{3 n}, \\
& \mathbb{V}\left(Y_{1}\right) \sim \frac{20}{9}+\frac{-4 \gamma+14 / 9}{n}-\frac{4 \ln n}{n} .
\end{aligned}
$$

Similarly, for $Y_{2}$, we obtain

$$
G(1, v)=\frac{v(v-4)}{3(v-2)}+\frac{2 v(v-1)}{3(v-2) n}+\frac{4 n^{v}}{\left(v^{2}-v-2\right) \Gamma(v-1) n^{2}},
$$

from which we derive the moments

$$
\begin{aligned}
& \mathbb{E}\left(Y_{2}\right) \sim \frac{5}{3}-\frac{8}{3 n}, \\
& \mathbb{V}\left(Y_{2}\right) \sim \frac{14}{9}+\frac{-4 \gamma+14 / 9}{n}-\frac{4 \ln n}{n} .
\end{aligned}
$$

Finally, the covariance is given by

$$
\operatorname{Cov}\left(Y_{1}, Y_{2}\right)=\frac{1}{9}-\frac{14}{9 n}+\mathcal{O}\left(\frac{\ln n}{n^{2}}\right)
$$

All these quantities can also be obtained by suitable integrals over previous expressions. Indeed, by the Euler-Maclaurin summation formula, we have

$$
\sum_{1 \leq j<k \leq n} \mathbb{E}\left(X_{k}\right)=\sum_{k=1}^{n}(k-1) \mathbb{E}\left(X_{k}\right) \sim \int_{0}^{1}(\alpha n-1) E(\alpha) n d \alpha-E(0) / 2+E(1) / 2,
$$


where $E(\alpha)$ is given by (11). Multiplying by $\frac{1}{n(n-1) / 2}$, this leads asymptotically to (14). Similarly, we obtain

$$
\sum_{1 \leq j<k \leq n} \mathbb{E}\left(X_{j}\right)=\sum_{j=1}^{n}(n-j) \mathbb{E}\left(X_{j}\right) \sim \int_{0}^{1}(n-\beta n) E(\beta) n d \beta-E(0) / 2+E(1) / 2 .
$$

Second moments and variances are derived in a similar manner. For the covariance, we have

$$
\begin{aligned}
\sum_{j=1}^{n-1} \sum_{k=j+1}^{n} \mathbb{E}\left(X_{k} X_{j}\right) & =\sum_{j=1}^{n} \sum_{k=j+1}^{n} \mathbb{E}\left(X_{k} X_{j}\right) \sim \int_{\beta=0}^{1} F(\beta) n d \beta-F(0) / 2+F(1) / 2, \text { with } \\
F(\beta) & =\int_{\alpha=\beta}^{1} E(\alpha, \beta) n d \alpha-E(\beta, \beta) / 2+E(1, \beta) / 2,
\end{aligned}
$$

and $E(\alpha, \beta)$ is given by (13). The aymptotic covariance (15) follows easily.

\section{The nUmber of MOVes in the Fisher-Yates ALGORIthm}

In this chapter, we consider the analogous generating functions $\phi_{n, k}^{(1)}(u)$ and (later) $\phi_{n, k, j}^{(2)}(u, v)$ for the Fisher-Yates algorithm. The explanations for the recursions are basically the same as for Sattolo's algorithm, the only difference being that an element can possibly be interchanged with itself (which is also counted as a move, to keep everything consistent).

First of all, we have the following recursions for the univariate generating functions $\phi_{n, k}^{(1)}(u)$ :

$$
\begin{gathered}
\phi_{n, n}^{(1)}(u)=\frac{u}{n}\left[1+\sum_{1 \leq k<n} \phi_{n-1, k}^{(1)}(u)\right], \quad n \geq 1, \\
\phi_{n, k}^{(1)}(u)=\frac{n-k}{n} u+\frac{k}{n} \phi_{k, k}^{(1)}(u) \quad 1 \leq k \leq n .
\end{gathered}
$$

The method to solve these recursions is the same as for the corresponding recursions in Section 2. We have

$$
\begin{aligned}
n \phi_{n, n}^{(1)}(u) & =u+u \sum_{1 \leq k<n} \phi_{n-1, k}^{(1)}(u) \\
& =u+u \sum_{1 \leq k<n}\left[\frac{n-1-k}{n-1} u+\frac{k}{n-1} \phi_{k, k}^{(1)}(u)\right] \\
& =u+\frac{(n-2) u^{2}}{2}+\frac{u}{n-1} \sum_{1 \leq k<n} k \phi_{k, k}^{(1)}(u)
\end{aligned}
$$

and thus

$$
n(n-1) \phi_{n, n}^{(1)}(u)=u(n-1)+\frac{(n-1)(n-2) u^{2}}{2}+u \sum_{1 \leq k<n} k \phi_{k, k}^{(1)}(u) .
$$

Now, we take differences:

$$
n(n-1) \phi_{n, n}^{(1)}(u)-(n-1)(n-2) \phi_{n-1, n-1}^{(1)}(u)=u+(n-2) u^{2}+u(n-1) \phi_{n-1, n-1}^{(1)}(u),
$$


from which it follows that

or

$$
\begin{aligned}
\frac{n !}{(n-2+u) !} \phi_{n, n}^{(1)}(u) & =\frac{(n-1) !}{(n-3+u) !} \phi_{n-1, n-1}^{(1)}(u)+\frac{\left(u+(n-2) u^{2}\right)(n-2) !}{(n-2+u) !} \\
& =\frac{1}{(u-1) !} \phi_{1,1}^{(1)}(u)+\sum_{k=0}^{n-2} \frac{\left(u+k u^{2}\right) k !}{(k+u) !} \\
& =\frac{u}{(u-1) !}+\frac{u(n-1) !(2+(n-1) u)}{(n-2+u) !(2-u)}-\frac{2 u}{(2-u)(u-1) !},
\end{aligned}
$$

$$
\phi_{n, n}^{(1)}(u)=-\frac{u^{2}(n-2+u) !}{(2-u)(u-1) ! n !}+\frac{u(2+(n-1) u)}{(2-u) n} .
$$

Finally, this gives us the desired explicit formula:

$$
\phi_{n, k}^{(1)}(u)=\frac{n-k}{n} u-\frac{u^{2}(k-2+u) !}{n(2-u)(u-1) !(k-1) !}+\frac{u(2+(k-1) u)}{(2-u) n} .
$$

Again, it is a routine task to get the expectation from it:

$$
\mathbb{E}\left(X_{k}\right)=\frac{n+2 k-2-H_{k-1}}{n},
$$

a result that has already appeared in Wilson's paper [10]. However, it is not more complicated to go one step further and compute the second factorial moment as well:

$$
\frac{8 k-8-H_{k-1}^{2}-6 H_{k-1}+H_{k-1}^{(2)}}{n} .
$$

The variance can be determined in the usual way from these two results. Again, we also give the grand average:

$$
\frac{1}{n} \sum_{1 \leq k \leq n} \phi_{n, k}^{(1)}(u)=\frac{u(n+1)}{2-u}-\frac{u}{2-u}\left(\begin{array}{c}
n+u-1 \\
n
\end{array}\right)
$$

Now we continue, as before, with the moves of two elements: not surprisingly (in view of the common structure of the two algorithms), the recursions are very similar to those for Sattolo's algorithm. For $1 \leq j<k<n$, we have

$$
\phi_{n, k, j}^{(2)}(u, v)=\frac{k(k-1)}{n(n-1)} \phi_{k, k, j}^{(2)}(u, v)+\sum_{l=k}^{n-1} \frac{l}{n(n-1)} u \phi_{l, j}^{(1)}(v)+\sum_{l=k}^{n-1} \frac{l}{n(n-1)} v \phi_{l, k}^{(1)}(u) .
$$

Furthermore, for $1 \leq j<n$,

$$
\phi_{n, n, j}^{(2)}(u, v)=\frac{u}{n}\left[\sum_{l=1}^{j-1} \phi_{n-1, j, l}^{(2)}(v, u)+\sum_{l=j+1}^{n-1} \phi_{n-1, l, j}^{(2)}(u, v)+v \phi_{n-1, j}^{(1)}(u)+\phi_{n-1, j}^{(1)}(v)\right] .
$$

The recursions can be justified in exactly the same way as in Section 2: the summands of the first recursion correspond to the cases "elements $k$ and $j$ are never chosen to move to the right during the first $n-k$ steps", "element $k$ moves to the right during these steps (before $j$ does)", "element $j$ moves to the right during these steps (before $k$ does)". In the latter two cases, one only needs to continue with the remaining moves of a single element.

In the second recursion, there is just a slight difference to Sattolo's algorithm: since $n$ is also allowed to be interchanged with itself, we obtain four summands. 
Now we can proceed as in Section 2, with the additional advantage that the cases $j=1$ and $j>1$ don't have to be distinguished. First of all, we calculate the sums $\sum_{l=k}^{n-1} \frac{l}{n(n-1)} u \phi_{l, j}^{(1)}(v)$ and $\sum_{l=k}^{n-1} \frac{l}{n(n-1)} v \phi_{l, k}^{(1)}(u)$ :

$$
\begin{aligned}
\sum_{l=k}^{n-1} \frac{l}{n(n-1)} u \phi_{l, j}^{(1)}(v) & =\sum_{l=k}^{n-1} \frac{u v}{(v-2) n(n-1)}\left[-2 n-2(v-1) j+v(l+1)+v\left(\begin{array}{c}
j+v-2 \\
j-1
\end{array}\right)\right] \\
& =\frac{u v(n-k)}{(v-2) n(n-1)}\left[\frac{(v-2)(n+k+1)-4(v-1) j}{2}+v\left(\begin{array}{c}
j+v-2 \\
j-1
\end{array}\right)\right]
\end{aligned}
$$

and

$$
\begin{aligned}
\sum_{l=k}^{n-1} \frac{l}{n(n-1)} v \phi_{l, k}^{(1)}(u) & =\sum_{l=k}^{n-1} \frac{u v}{(u-2) n(n-1)}\left[-2 n-2(u-1) k+u(l+1)+u\left(\begin{array}{c}
k+u-2 \\
k-1
\end{array}\right)\right] \\
& =\frac{u v(n-k)}{(u-2) n(n-1)}\left[\frac{(u-2) n-(3 u-2) k+u-2}{2}+u\left(\begin{array}{c}
k+u-2 \\
k-1
\end{array}\right)\right] .
\end{aligned}
$$

It follows that

$$
\begin{aligned}
& \phi_{n, k, j}^{(2)}(u, v)= \frac{k(k-1)}{n(n-1)} \phi_{k, k, j}^{(2)}(u, v)+\frac{u v(n-k)}{(u-2)(v-2) n(n-1)} \\
& \quad \times\left[\frac{(u-2)((v-2)(n+k+1)-4(v-1) j)}{2}+\frac{(v-2)((u-2) n-(3 u-2) k+u-2)}{2}\right. \\
&\left.\quad+v(u-2)\left(\begin{array}{c}
j+v-2 \\
j-1
\end{array}\right)+u(v-2)\left(\begin{array}{c}
k+u-2 \\
k-1
\end{array}\right)\right] \\
&=\frac{k(k-1)}{n(n-1)} \phi_{k, k, j}^{(2)}(u, v)+\frac{u v(n-k)}{n(n-1)} \\
& \quad \times\left[n-k-2 j+1-\frac{2 j}{v-2}-\frac{2 k}{u-2}+\frac{v}{v-2}\left(\begin{array}{c}
j+v-2 \\
j-1
\end{array}\right)+\frac{u}{u-2}\left(\begin{array}{c}
k+u-2 \\
k-1
\end{array}\right)\right] .
\end{aligned}
$$

So we have, for $1 \leq j<n$,

$$
\phi_{n, n, j}^{(2)}(u, v)=\frac{u}{n}\left[\sum_{l=1}^{j-1} \frac{j(j-1)}{(n-1)(n-2)} \phi_{j, j, l}^{(2)}(v, u)+\sum_{l=j+1}^{n-1} \frac{l(l-1)}{(n-1)(n-2)} \phi_{l, l, j}^{(2)}(u, v)+X_{1}\right],
$$

where $X_{1}$ is used as an abbreviation for

$$
\begin{aligned}
X_{1}:= & \sum_{l=1}^{j-1} \frac{u v(n-j-1)}{(n-1)(n-2)}\left[n-j-2 l-\frac{2 l}{u-2}-\frac{2 j}{v-2}+\frac{u}{u-2}\left(\begin{array}{c}
l+u-2 \\
l-1
\end{array}\right)+\frac{v}{v-2}\left(\begin{array}{c}
j+v-2 \\
j-1
\end{array}\right)\right] \\
& +\sum_{l=j+1}^{n-1} \frac{u v(n-l-1)}{(n-1)(n-2)}\left[n-l-2 j-\frac{2 j}{v-2}-\frac{2 l}{u-2}+\frac{v}{v-2}\left(\begin{array}{c}
j+v-2 \\
j-1
\end{array}\right)+\frac{u}{u-2}\left(\begin{array}{c}
l+u-2 \\
l-1
\end{array}\right)\right] \\
& +\frac{v}{n-1}\left[(u+1) n-2(u+2) j-\frac{2 j}{v-2}-\frac{4 j}{u-2}+\frac{u^{2}}{u-2}\left(\begin{array}{c}
j+u-2 \\
j-1
\end{array}\right)+\frac{v}{v-2}\left(\begin{array}{c}
j+v-2 \\
j-1
\end{array}\right)\right] .
\end{aligned}
$$


Again, we calculate the sums and simplify to obtain

$$
\begin{aligned}
X_{1}= & \frac{v}{3(u-2)(v-2)(n-1)(n-2)}\left[u(u-3)(v-2) n^{3}-3 u(u-2)(v-1) n^{2} j\right. \\
& -3(v-2)\left(u^{2}-4 u+2\right) n^{2}+3(u-2)(5 u-2)(v-1) n j-\left(u^{2}+6 u-12\right)(v-2) n \\
& +u(2 u v-u-3 v) j^{3}-3 u(4 u v-5 u-6 v+6) j^{2}-\left(2 u^{2} v+8 u^{2}-21 u v+6 u+24 v-24\right) j \\
& +\frac{3(j-1) u\left(u^{2}-j+1\right)(v-2)}{u+1}\left(\begin{array}{c}
j+u-2 \\
j-1
\end{array}\right)+\frac{3 u(v-2)(n-1)(n-2)}{u+1}\left(\begin{array}{c}
n+u-2 \\
n-1
\end{array}\right) \\
& \left.+\frac{3(u-2) v\left(u n^{2}-5 u n+2 n-u j^{2}+3 u j+4 u-4\right)}{2}\left(\begin{array}{c}
j+v-2 \\
j-1
\end{array}\right)\right] .
\end{aligned}
$$

Now, we perform the substitution $n(n-1) \phi_{n, n, j}^{(2)}(u, v)=R_{n, j}(u, v)$ (as in Section 2), which yields

$$
(n-2) R_{n, j}(u, v)=u \sum_{l=1}^{j-1} R_{j, l}(v, u)+u \sum_{l=j+1}^{n-1} R_{l, j}(u, v)+X_{2},
$$

where $X_{2}:=u(n-1)(n-2) X_{1}$. Taking differences, it follows that

$$
\begin{aligned}
& (n-2) R_{n, j}(u, v)-(n-3) R_{n-1, j}(u, v) \\
& \quad=u \sum_{l=1}^{j-1} R_{j, l}(v, u)+u \sum_{l=j+1}^{n-1} R_{l, j}(u, v)-u \sum_{l=1}^{j-1} R_{j, l}(v, u)-u \sum_{l=j+1}^{n-2} R_{l, j}(u, v)+X_{3} \\
& =u R_{n-1, j}(u, v)+X_{3}
\end{aligned}
$$

or

$$
\frac{(n-2) !}{(n-3+u) !} R_{n, j}(u, v)=\frac{(n-3) !}{(n-4+u) !} R_{n-1, j}(u, v)+\frac{(n-3) !}{(n-3+u) !} X_{3},
$$

with

$$
\begin{array}{r}
X_{3}:=\frac{u v\left[u(u-3) n^{2}-\left(3 u^{2}-11 u+4\right) n+(u-1)(u-6)\right]}{u-2}-\frac{2 u v(v-1)(u n-3 u+1) j}{v-2} \\
+\frac{u^{2} v(n-2)}{u-2}\left(\begin{array}{c}
n+u-3 \\
n-2
\end{array}\right)+\frac{u v^{2}(u n-3 u+1)}{v-2}\left(\begin{array}{c}
j+v-2 \\
j-1
\end{array}\right) .
\end{array}
$$

Now we set $S_{n, j}(u, v)=\frac{(n-2) !}{(n-3+u) !} R_{n, j}(u, v)=\frac{n !}{(n-3+u) !} \phi_{n, n, j}^{(2)}(u, v)$ again and obtain

$$
\begin{aligned}
S_{n, j}(u, v)=S_{n-1, j}(u, v)+\frac{u^{2} v}{(u-2)(u-1) !}+\frac{u^{2} v(u-3)}{u-2} \cdot \frac{(n-1) !}{(n-3+u) !} \\
+\frac{u v}{v-2}\left[u v\left(\begin{array}{c}
j+v-2 \\
j-1
\end{array}\right)-2 u(v-1) j+2(v-2)\right] \cdot \frac{(n-2) !}{(n-3+u) !} \\
\quad+\frac{u v(u-1)}{v-2}\left[2(v-1) j-(v-2)-v\left(\begin{array}{c}
j+v-2 \\
j-1
\end{array}\right)\right] \cdot \frac{(n-3) !}{(n-3+u) !} .
\end{aligned}
$$

Summing this for $n=j+2, \ldots, N$ yields

$$
S_{N, j}(u, v)=S_{j+1, j}(u, v)+\frac{u^{2} v(N-j-1)}{(u-2)(u-1) !}+\frac{u^{2} v}{u-2}\left[\frac{(j+1) !}{(j+u-2) !}-\frac{N !}{(N-3+u) !}\right]
$$




$$
\begin{aligned}
& +\frac{u v}{(u-2)(v-2)}\left[u v\left(\begin{array}{c}
j+v-2 \\
j-1
\end{array}\right)-2 u(v-1) j+2(v-2)\right]\left[\frac{j !}{(j+u-2) !}-\frac{(N-1) !}{(N-3+u) !}\right] \\
& +\frac{u v}{v-2}\left[2(v-1) j-(v-2)-v\left(\begin{array}{c}
j+v-2 \\
j-1
\end{array}\right)\right]\left[\frac{(j-1) !}{(j+u-2) !}-\frac{(N-2) !}{(N-3+u) !}\right] .
\end{aligned}
$$

As in Section 2, it remains to compute $S_{j+1, j}(u, v)$, or equivalently $R_{j+1, j}(u, v)$ :

$$
\begin{aligned}
(j-1) R_{j+1, j}(u, v)= & u \sum_{l=1}^{j-1} R_{j, l}(v, u)+\frac{u v(j-1)}{(u-2)(v-2)}\left[(u+1)(u-2)(v-2)-\left(u^{2} v-2 u^{2}+u v-2 v\right) j\right] \\
& +\frac{(j-1) u^{3} v}{u-2}\left(\begin{array}{c}
j+u-2 \\
j-1
\end{array}\right)+\frac{(j-1) u v^{2}}{v-2}\left(\begin{array}{c}
j+v-2 \\
j-1
\end{array}\right),
\end{aligned}
$$

which can be rewritten as

$$
\begin{aligned}
\frac{(j-1)(j-2+u) !}{(j-1) !} & S_{j+1, j}(u, v)=u \sum_{l=1}^{j-1} \frac{(j-3+v) !}{(j-2) !} S_{j, l}(v, u) \\
& +\frac{u v(j-1)}{(u-2)(v-2)}\left[(u+1)(u-2)(v-2)-\left(u^{2} v-2 u^{2}+u v-2 v\right) j\right] \\
& +\frac{(j-1) u^{3} v}{u-2}\left(\begin{array}{c}
j+u-2 \\
j-1
\end{array}\right)+\frac{(j-1) u v^{2}}{v-2}\left(\begin{array}{c}
j+v-2 \\
j-1
\end{array}\right),
\end{aligned}
$$

which in turn reduces to

$$
\begin{aligned}
S_{j+1, j}(u, v)=\frac{u(j-3+v) !}{(j-2+u) !} \sum_{l=1}^{j-1} S_{j, l}(v, u)+\frac{u v(j-1) !}{(u-2)(v-2)(j-2+u) !} \\
\quad \times\left[(u+1)(u-2)(v-2)-\left(u^{2} v-2 u^{2}+u v-2 v\right) j\right] \\
\quad+\frac{u^{3} v}{(u-2)(u-1) !}+\frac{(j-1) ! u v^{2}}{(v-2)(j-2+u) !}\left(\begin{array}{c}
j+v-2 \\
j-1
\end{array}\right) .
\end{aligned}
$$

Now we express $S_{j, l}(v, u)$ in terms of $S_{l+1, l}(v, u)$ again:

$$
\begin{aligned}
S_{j, l}(v, u)= & S_{l+1, l}(v, u)+\frac{u v^{2}(j-l-1)}{(v-2)(v-1) !}+\frac{u v^{2}}{v-2}\left[\frac{(l+1) !}{(l+v-2) !}-\frac{j !}{(j-3+v) !}\right] \\
& +\frac{u v}{(u-2)(v-2)}\left[u v\left(\begin{array}{c}
l+u-2 \\
l-1
\end{array}\right)-2 v(u-1) l+2(u-2)\right]\left[\frac{l !}{(l+v-2) !}-\frac{(j-1) !}{(j-3+v) !}\right] \\
& +\frac{u v}{u-2}\left[2(u-1) l-(u-2)-u\left(\begin{array}{c}
l+u-2 \\
l-1
\end{array}\right)\right]\left[\frac{(l-1) !}{(l+v-2) !}-\frac{(j-2) !}{(j-3+v) !}\right] \\
= & S_{l+1, l}(v, u)+\frac{u v^{2}(j-1)}{(v-2)(v-1) !}-\frac{u v\left(v j^{2}-(v-2) j-v\right)(j-2) !}{(v-2)(j+v-3) !} \\
& +l \cdot\left[-\frac{u v^{2}}{(v-2)(v-1) !}+\frac{2 u v(u-1)(v j-2 v+2)(j-2) !}{(u-2)(v-2)(j+v-3) !}\right] \\
& -\frac{u v(l-1) !}{(l+v-2) !}+\frac{2 u v(2 u v-u-2 v) l !}{(u-2)(v-2)(l+v-2) !}-\frac{u^{2} v^{2}(l+1) !}{(u-2)(v-2)(l+v-2) !} \\
& -\frac{u^{2} v(j v-2 v+2)(j-2) !}{(u-2)(v-2)(j+v-3) !}\left(\begin{array}{c}
l+u-2 \\
l-1
\end{array}\right)+\frac{u^{2} v^{2}(l+u-1) !}{(u-2)(v-2)(u-1) !(l+v-2) !}
\end{aligned}
$$




$$
-\frac{u^{2} v(u v-2)(l+u-2) !}{(u-2)(v-2)(u-1) !(l+v-2) !} .
$$

Summing this for $l=1, \ldots, j-1$ yields

$$
\begin{aligned}
\sum_{l=1}^{j-1} S_{j, l}(v, u)= & \sum_{l=1}^{j-1} S_{l+1, l}(v, u)+\frac{u v^{2}(j-1)^{2}}{(v-2)(v-1) !}-\frac{u v\left(v j^{2}-(v-2) j-v\right)(j-1) !}{(v-2)(j+v-3) !} \\
& +\frac{j(j-1)}{2}\left[-\frac{u v^{2}}{(v-2)(v-1) !}+\frac{2 u v(u-1)(v j-2 v+2)(j-2) !}{(u-2)(v-2)(j+v-3) !}\right] \\
& -\frac{u v}{v-2}\left[\frac{1}{(v-2) !}-\frac{(j-1) !}{(j+v-3) !}\right] \\
& +\frac{2 u v(2 u v-u-2 v)}{(u-2)(v-2)(v-3)}\left[\frac{1}{(v-2) !}-\frac{j !}{(j+v-3) !}\right] \\
& -\frac{u^{2} v^{2}}{(u-2)(v-2)(v-4)}\left[\frac{2}{(v-2) !}-\frac{(j+1) !}{(j+v-3) !}\right] \\
- & \frac{u^{2} v(j v-2 v+2)(j-2) !}{(u-2)(v-2)(j+v-3) !}(j+u-2) \\
+ & \frac{u^{2} v^{2}}{(u-2)(v-2)(u-1) !(v-u-2)}\left[\frac{u !}{(v-2) !}-\frac{(j+u-1) !}{(j+v-3) !}\right] \\
& -\frac{u^{2} v(u v-2)}{(u-2)(v-2)(u-1) !(v-u-1)}\left[\frac{(u-1) !}{(v-2) !}-\frac{(j+u-2) !}{(j+v-3) !}\right] .
\end{aligned}
$$

Now it follows that

$$
\frac{(j-2+u) !}{u(j-3+v) !} S_{j+1, j}(u, v)=\sum_{l=1}^{j-1} S_{l+1, l}(v, u)+Q_{j}(u, v),
$$

where

$$
\begin{aligned}
Q_{j}(u, v) & =\frac{v^{2}\left(u j^{2}-(3 u-2) j+4 u+2 v-4\right)}{2(v-2)(v-1) !}+\frac{u v}{(u-v+1)(u-v+2)(v-2)(v-3)(v-4)(v-1) !} \\
& \times\left[u v^{4}-12 u v^{3}+5 v^{4}+u^{2} v^{2}-11 u^{2} v+52 u v^{2}-38 v^{3}+4 u^{2}-67 u v+91 v^{2}+20 u-82 v+24\right] \\
& +\frac{v(2 u v-u+v-2)(j-1) !}{(v-2)(j+v-3) !}-\frac{2 v\left(u^{2} v^{2}-2 u^{2} v+u v^{2}+2 u^{2}-6 u v-v^{2}+3 u+3 v\right) j !}{(u-2)(v-2)(v-3)(j+v-3) !} \\
& +\frac{u v^{2}(u+v-4)(j+1) !}{(u-2)(v-2)(v-4)(j+v-3) !}+\frac{u v\left(u^{2}-2 u v+u-v+1\right)(j+u-2) !}{(u-2)(u-v+1)(u-1) !(j+v-3) !} \\
& +\frac{u v^{2}(j+u-1) !}{(u-2)(u-v+2)(u-1) !(j+v-3) !} .
\end{aligned}
$$

Elimination yields

$\frac{(j-1+u) !}{u(j-2+v) !} S_{j+2, j+1}(u, v)-\frac{(j-2+u) !}{u(j-3+v) !} S_{j+1, j}(u, v)=S_{j+1, j}(v, u)+Q_{j+1}(u, v)-Q_{j}(u, v)$,

and we have

$P_{j}(u, v):=Q_{j+1}(u, v)-Q_{j}(u, v)$ 


$$
\begin{aligned}
= & \frac{v^{2}(u j-u+1)}{(v-2)(v-1) !}-\frac{v(2 u v-u+v-2)(j-1) !}{(j+v-2) !} \\
& +\frac{2 v\left(u^{2} v^{2}-2 u^{2} v+u v^{2}+2 u^{2}-6 u v-v^{2}+3 u+3 v\right) j !}{(u-2)(v-2)(j+v-2) !}-\frac{u v^{2}(u+v-4)(j+1) !}{(u-2)(v-2)(j+v-2) !} \\
& +\frac{u v\left(u^{2}-2 u v+u-v+1\right)(j+u-2) !}{(u-2)(u-1) !(j+v-2) !}+\frac{u v^{2}(j+u-1) !}{(u-2)(u-1) !(j+v-2) !} .
\end{aligned}
$$

Writing $C_{j}(u, v)=\frac{(j-2+u) !}{u(j-3+v) !}$, we obtain

$$
C_{j+1}(u, v) S_{j+2, j+1}(u, v)-C_{j}(u, v) S_{j+1, j}(u, v)=S_{j+1, j}(v, u)+P_{j}(u, v) .
$$

Interchanging $u$ and $v$ and combining the two equations, we arrive at

$$
\begin{aligned}
& C_{j+1}(v, u) C_{j+2}(u, v) S_{j+3, j+2}(u, v)-C_{j+1}(u, v)\left(C_{j}(v, u)+C_{j+1}(v, u)\right) S_{j+2, j+1}(u, v) \\
& +\left(C_{j}(u, v) C_{j}(v, u)-1\right) S_{j+1, j}(u, v)=C_{j+1}(v, u) P_{j+1}(u, v)-C_{j}(v, u) P_{j}(u, v)+P_{j}(v, u) .
\end{aligned}
$$

After some simplifications, we see that this is equivalent to

$$
\begin{aligned}
(j+u)(j+u-1) S_{j+3, j+2}(u, v)-(j+u-1)(2 j+u+v-3) S_{j+2, j+1}(u, v) & \\
& +(j-2)(j+u+v-2) S_{j+1, j}(u, v)=L_{j}(u, v)
\end{aligned}
$$

for $j \geq 1$, where $L_{j}(u, v)$ is given by

$$
\begin{aligned}
L_{j}(u, v)= & \frac{u^{2} v\left(v(u+2) j+u^{2}-u v+2 u-3 v+1\right)}{(u-2)(u-1) !}+\frac{2 u v(j-1) !}{(u-2)(v-2)(j+u-2) !} \\
& \times\left[-2 u v(u+v-4) j^{2}+\left(3 u^{3} v-3 u^{3}-8 u^{2} v+4 u v^{2}+6 u^{2}-10 u v-3 v^{2}+9 u+9 v\right) j\right. \\
& -(u-2)(v-2)(u+1)(u+v-2)] \\
& +\frac{u v^{2}\left(u(v+2) j-u^{2} v+u v^{2}-u^{2}+u v-2 u+v+1\right)(j+v-2) !}{(v-2)(j+u-2) !(v-1) !} .
\end{aligned}
$$

Setting $S_{j+1, j}(u, v)=s_{j-1}$ for $j \geq 1$, we obtain the recurrence

$$
(j+u+1)(j+u) s_{j+2}-(j+u)(2 j+u+v-1) s_{j+1}+(j-1)(j+u+v-1) s_{j}=h_{j}
$$

with

$$
\begin{aligned}
h_{j}= & \frac{u^{2} v\left(v(u+2) j+(u+1)^{2}-v\right)}{(u-2)(u-1) !}-\frac{4 u^{2} v^{2}(u+v-4)(j+2) !}{(u-2)(v-2)(j+u-1) !} \\
& +\frac{6 u v\left(u^{3} v-2 u^{2} v+2 u v^{2}-u^{3}+2 u^{2}-6 u v-v^{2}+3 u+3 v\right)(j+1) !}{(u-2)(v-2)(j+u-1) !} \\
& -\frac{2 u v(u+1)(u+v-2) j !}{(j+u-1) !} \\
& +\frac{u^{2} v^{2}(v+2)(j+v) !}{(v-2)(j+u-1) !(v-1) !}-\frac{u v^{2}(u-1)(u+1)(v+1)(j+v-1) !}{(v-2)(j+u-1) !(v-1) !}
\end{aligned}
$$

for $j \geq 0$. The initial values are given by $s_{0}=\frac{u v(u+1)}{(u-1) !}$ and $s_{1}=\frac{v(u+1)(u+v+1)}{(u-1) !}$. The explicit solution to this recurrence (determined by means of computer algebra again) is now found 
to be

$$
\begin{aligned}
s_{j}= & -\frac{u^{2} v(v j+2 u-v+2)}{(u-2)(v-2)(u-1) !}+\frac{u v j !}{(u-2)(v-2)(u+v-3)(j+u-1) !} \\
& \times\left[u v(u+v-3) j^{2}-\left(2 u^{3} v-2 u^{3}-7 u^{2} v+u v^{2}+4 u^{2}-3 u v-2 v^{2}+6 u+6 v\right) j\right. \\
& \left.-\left(u^{3} v-u^{2} v^{2}+3 u v^{2}-4 u^{2}-9 u v+8 u-4 v+12\right)\right] \\
& -\frac{u v^{2}(u+v-3)\left(u j-u^{2}+u v+u+2\right)(j+v-1) !}{(u-2)(v-2)(u+v-3)(j+u-1) !(v-1) !} \\
& +\frac{u^{2} v^{2}\left(u^{2}+v^{2}-u-v-2\right)(j+u+v-2) !}{(u-2)(v-2)(u+v-3)(j+u-1) !(u+v-2) !} .
\end{aligned}
$$

It follows that

$$
\begin{aligned}
S_{j+1, j}(u, v)= & -\frac{u^{2} v(v j+2 u-2 v+2)}{(u-2)(v-2)(u-1) !}+\frac{u v(j-1) !}{(u-2)(v-2)(u+v-3)(j+u-2) !} \\
& \times\left[u v(u+v-3) j^{2}-\left(2 u^{3} v-2 u^{3}-5 u^{2} v+3 u v^{2}+4 u^{2}-9 u v-2 v^{2}+6 u+6 v\right) j\right. \\
& +(u-2)(v-2)(u+1)(u+v-3)] \\
& -\frac{u v^{2}(u+v-3)\left(u j-u^{2}+u v+2\right)(j+v-2) !}{(u-2)(v-2)(u+v-3)(j+u-2) !(v-1) !} \\
& +\frac{u^{2} v^{2}\left(u^{2}+v^{2}-u-v-2\right)(j+u+v-3) !}{(u-2)(v-2)(u+v-3)(j+u-2) !(u+v-2) !}
\end{aligned}
$$

and

$$
\begin{aligned}
S_{n, j}(u, v)= & \frac{u^{2} v((v-2) n-2(v-1) j+v-2 u)}{(u-2)(v-2)(u-1) !}+\frac{u^{2} v(j-1) !}{(j+u-2) !}\left[1-\frac{2(u-1)(u-3)(v-1) j}{(u-2)(v-2)(u+v-3)}\right] \\
& +\frac{u^{2} v^{2}(u-v-1)(j+v-2) !}{(u-2)(v-2)(v-1) !(j+u-2) !}+\frac{u^{2} v^{2}\left(u^{2}+v^{2}-u-v-2\right)(j+u+v-3) !}{(u-2)(v-2)(u+v-3)(j+u-2) !(u+v-2) !} \\
& -\frac{u v(n-2) !}{(n+u-3) !}\left[\frac{u n^{2}}{u-2}-\frac{(2 u(v-1) j+(u-2)(v-2)) n}{(u-2)(v-2)}+\frac{4(u-1)(v-1) j-u(v-2)}{(u-2)(v-2)}\right] \\
& -\frac{u v^{2}(u n-2 u+2)(n-2) !}{(u-2)(v-2)(n+u-3) !}\left(\begin{array}{c}
j+v-2 \\
j-1
\end{array}\right) .
\end{aligned}
$$

Now, we obtain an expression for $\phi_{n, n, j}^{(2)}(u, v)=\frac{(n-3+u) !}{n !} S_{n, j}(u, v)$ :

$$
\begin{aligned}
\phi_{n, n, j}^{(2)}(u, v)= & \frac{(n-3+u) !}{n !}\left[\frac{u^{2} v((v-2) n-2(v-1) j+v-2 u)}{(u-2)(v-2)(u-1) !}\right. \\
& +\frac{u^{2} v(j-1) !}{(j+u-2) !}\left(1-\frac{2(u-1)(u-3)(v-1) j}{(u-2)(v-2)(u+v-3)}\right)+\frac{u^{2} v^{2}(u-v-1)(j+v-2) !}{(u-2)(v-2)(v-1) !(j+u-2) !} \\
& \left.+\frac{u^{2} v^{2}\left(u^{2}+v^{2}-u-v-2\right)(j+u+v-3) !}{(u-2)(v-2)(u+v-3)(j+u-2) !(u+v-2) !}\right] \\
& -\frac{u v}{(n-1) n}\left[\frac{u n^{2}}{u-2}-\frac{(2 u(v-1) j+(u-2)(v-2)) n}{(u-2)(v-2)}+\frac{4(u-1)(v-1) j-u(v-2)}{(u-2)(v-2)}\right]
\end{aligned}
$$




$$
-\frac{u v^{2}(u n-2 u+2)}{(u-2)(v-2)(n-1) n}\left(\begin{array}{c}
j+v-2 \\
j-1
\end{array}\right) .
$$

Finally, we get the following explicit formula for $\phi_{n, k, j}^{(2)}(u, v)$ (which holds for all values of $j$ in this case):

Theorem 4.1. For $1 \leq j<k \leq n$, we have

$$
\begin{aligned}
\phi_{n, k, j}^{(2)}(u, v)= & \frac{(k-3+u) !}{(k-2) ! n(n-1)}\left[\frac{u^{2} v(j-1) !}{(j+u-2) !}\left(1-\frac{2(u-1)(u-3)(v-1) j}{(u-2)(v-2)(u+v-3)}\right)\right. \\
& \left.+\frac{u^{2} v^{2}(u-v-1)(j+v-2) !}{(u-2)(v-2)(v-1) !(j+u-2) !}+\frac{u^{2} v^{2}\left(u^{2}+v^{2}-u-v-2\right)(j+u+v-3) !}{(u-2)(v-2)(u+v-3)(j+u-2) !(u+v-2) !}\right] \\
& +\frac{u^{2} v}{(u-2) n(n-1)}\left(\begin{array}{c}
k+u-2 \\
k-1
\end{array}\right)\left[n-k+\frac{k(k-1)}{k+u-2}-\frac{(k-1)(2(v-1) j+2 u-v)}{(v-2)(k+u-2)}\right] \\
& +\frac{u v^{2}((u-2) n-2(u-1)(k-1))}{(u-2)(v-2) n(n-1)}\left(\begin{array}{c}
j+v-2 \\
j-1
\end{array}\right)+u v-\frac{2 u v(u-1) k}{(u-2)(n-1)} \\
& -\frac{2 u v(j v-j-v+2)}{(v-2)(n-1)}+\frac{4 u v(u-1)(v-1) j(k-1)}{(u-2)(v-2) n(n-1)}+\frac{u^{2} v}{(u-2) n(n-1)} .
\end{aligned}
$$

Again, the correct formulæ arise if we set $u=1$ or $v=1$.

Of course, we can once more deduce all sorts of corollaries from this formula. For instance, the covariance can be determined from

$$
\begin{aligned}
\frac{\partial^{2}}{\partial u \partial v} & \left.\phi_{n, k, j}^{(2)}(u, v)\right|_{u=1, v=1}=\frac{1}{n(n-1)}\left[H_{j-1} H_{k-1}-(n+2 j-3) H_{k-1}\right. \\
& -\frac{\left(k n-n-12 k+2 k^{2}+11\right) H_{j-1}}{k-1}-2 H_{j-1}^{(2)}+H_{j-1}^{2} \\
& \left.+\frac{-18+16 j+22 k+5 n-7 k n+2 k^{2} n-6 k^{2}+4 j k^{2}-18 j k-2 n j+k n^{2}-n^{2}+2 n j k}{k-1}\right] .
\end{aligned}
$$

We also give the grand average in this case:

$$
\begin{aligned}
G_{n}(u, v) & =\frac{1}{\left(\begin{array}{c}
n \\
2
\end{array}\right)} \sum_{1 \leq j<k \leq n} \phi_{n, k, j}^{(2)}(u, v) \\
& =-\frac{(-u-3+v)(n v+2 u v-2 v-3 n-2 u+2+n u)(n+1)}{3(u-2)(v-2)(u+v-3)(n-1) n} \\
& +\frac{2 u^{2} v(u+v-1)\left(u^{2}+v^{2}-u-v-2\right)(n+u+v-2) !}{(u-2)(v-2)(u+v-3)(u+v) ! n(n-1) n !} \\
& +\frac{2 u^{3} v(-u-3+v)(n+u) !}{(u+2)(u-2)(u+1) !(v-2) n(n-1) n !} \\
& -\frac{2 u v(u v+2)(n+v) !}{(u-2)(v-2)(v+2)(v+1)(v-1) ! n(n-1) n !} .
\end{aligned}
$$


The following quantities can be computed immediately (and the grand averages of mean, variance and covariance follow once more):

$$
\begin{aligned}
\left.\frac{\partial}{\partial u} G_{n}(u, v)\right|_{u=1, v=1} & =\frac{8 n^{2}-21 n+1-6(n-1) H_{n}}{6(n-1) n} \\
\left.\frac{\partial}{\partial v} G_{n}(u, v)\right|_{u=1, v=1} & =\frac{4 n^{2}-15 n-1-6(n-1) H_{n}}{6(n-1) n} \\
\left.\frac{\partial^{2}}{\partial u^{2}} G_{n}(u, v)\right|_{u=1, v=1} & =\frac{16 n^{2}-49 n+1-6(n-1) H_{n}^{2}+6(n-1) H_{n}^{(2)}-30(n-1) H_{n}}{6(n-1) n} \\
\left.\frac{\partial^{2}}{\partial v^{2}} G_{n}(u, v)\right|_{u=1, v=1} & =\frac{8 n^{2}-23 n-1-6(n-1) H_{n}^{2}+6(n-1) H_{n}^{(2)}-18(n-1) H_{n}}{6(n-1) n} \\
\left.\frac{\partial^{2}}{\partial u \partial v} G_{n}(u, v)\right|_{u=1, v=1} & =\frac{n^{2}-9 n+2 H_{n}^{2}-2 H_{n}^{(2)}-2(2 n-3) H_{n}}{(n-1) n} .
\end{aligned}
$$

\section{Asymptotics for the moves in the Fisher-Yates Algorithm}

The asymptotic analysis can be performed along the same lines as in Section 3. This time, we have to consider six terms in $\phi_{n, k, j}^{(2)}$. However, it is easily seen that we can neglect the second one and we are left with the following:

Theorem 5.1. $\phi_{n, k, j}^{(2)}(u, v) \sim F(u, v)$, where $F(u, v)=Y_{1}+Y_{3}+Y_{4}+Y_{5}+Y_{6}$ and the summands are given by

$$
\begin{aligned}
Y_{1} & =-\frac{1}{n} \frac{\alpha^{u-1} u^{2} v \beta^{2-u}\left(2 u^{2} v-2 u^{2}-8 u v+8 v u+6 v-6\right)}{(u-2)(v-2)(u+v-3) n}, \\
Y_{3} & =\frac{n^{u+v} \alpha^{u-1} u^{2} v^{2}\left(u^{2}+v^{2}-u-v-2\right) \beta^{v-1}}{(u-2)(v-2)(u+v-3) \Gamma(u+v-1) n^{4}}, \\
Y_{4} & =-\frac{n^{u} u^{2} v \alpha^{u-2}(2 \beta \alpha v+2 \alpha-\alpha v-2 \beta \alpha)}{(u-2) \Gamma(u)(v-2) n^{2}}, \\
Y_{5} & =-\frac{n^{v} u v^{2}(-u+2+2 \alpha u-2 \alpha) \beta^{v-1}}{(u-2)(v-2) \Gamma(v) n^{2}}, \\
Y_{6} & =\frac{u v(-u+2+2 \alpha u-2 \alpha)(-v+2+2 \beta v-2 \beta)}{(v-2)(u-2)}+\frac{2 u v N}{(2-v)(2-u)}, \\
N & =-3 \beta u v+3 \beta u+4 \beta v+4-2 u+u v-4 \beta-2 v-\alpha u v \\
& -2 \beta \alpha v-2 \beta \alpha u+\alpha v+2 \alpha u-2 \alpha+2 \beta \alpha u v .
\end{aligned}
$$

We note that the first term of $Y_{6}$ reproduces $\psi_{\alpha, \beta}^{2}(u, v)$, as given by (4): hence, the dominant asymptotic behaviour is the same as in the analysis of Sattolo's algorithm. Again, we can also calculate the asymptotics of the moments: we have

$$
F(u, 1)=-\frac{u(-u+2+2 \alpha u-2 \alpha)}{(u-2)}+\frac{u}{n}+\frac{u^{2} n^{u} \alpha^{u-1}}{(u-2) \Gamma(u) n^{2}},
$$

which leads to

$$
\mathbb{E}\left(X_{k}\right) \sim 1+2 \alpha+\frac{-\gamma-2-\ln \alpha}{n}-\frac{\ln n}{n},
$$




$$
\begin{aligned}
\mathbb{V}\left(X_{k}\right) & \sim 6 \alpha-4 \alpha^{2}+\frac{-6-5 \gamma-\gamma^{2}-2 \gamma \ln \alpha-5 \ln \alpha+8 \alpha+4 \gamma \alpha+4 \alpha \ln \alpha+\pi^{2} / 6-\ln ^{2} \alpha}{n} \\
& +\frac{(-2 \ln \alpha-5+4 \alpha-2 \gamma) \ln n}{n} .
\end{aligned}
$$

The covariance is given by

$$
\operatorname{Cov}\left(X_{k}, X_{j}\right)=\frac{4 \beta(\alpha-2)}{n}+\mathcal{O}\left(\frac{\ln n}{n^{2}}\right) .
$$

Finally we move to the asymptotics for the grand average generating function. Again, we denote by $Y_{1}$ and $Y_{2}$ the random variables related to $u$ and $v$ respectively. Proceeding as before, we obtain the following limiting joint distribution function:

Theorem 5.2. The grand average generating function $G_{n}(u, v)=\frac{1}{\left(\begin{array}{c}n \\ 2\end{array}\right)} \sum_{1 \leq j<k \leq n} \phi_{n, k, j}^{(2)}(u, v)$ is asymptotically equal to $G(u, v)$, where

$$
\begin{aligned}
G(u, v) & :=G_{1}+G_{2}+G_{3}+G_{4}, \\
G_{1} & :=\frac{2 n^{u+v}\left(u^{2}+v^{2}-u-v-2\right)(u+v-1) u^{2} v}{(u-2)(v-2)(u+v-3) \Gamma(u+v+1) n^{4}}, \\
G_{2} & :=-\frac{-u+v-3}{3(u-2)(v-2)}-\frac{2(-u+v-3)(-2+u v))}{3(u-2)(v-2)(u+v-3) n}, \\
G_{3} & :=\frac{2 n^{u} u^{3} v(-u+v-3)}{(u-2) \Gamma(u+3)(v-2) n^{2}}, \\
G_{4} & :=\frac{2 n^{v} u v(u v+2)}{(u-2)(v+1) \Gamma(v)\left(v^{2}-4\right) n^{2}} .
\end{aligned}
$$

Now, setting $v=1$, we obtain

$$
G(u, 1)=-\frac{u+2}{3(u-2)}-\frac{u^{2}+4 u+4}{3(u-2) n}+\frac{2 u^{2} n^{u}}{\left(u^{2}-u-2\right) \Gamma(u) n^{2}},
$$

from which we derive the moments

$$
\begin{aligned}
\mathbb{E}\left(Y_{1}\right) & \sim \frac{4}{3}+\frac{5 / 2-\gamma}{3 n}-\frac{\ln n}{n}, \\
\mathbb{V}\left(Y_{1}\right) & \sim \frac{20}{9}+\frac{1-10 \gamma / 3+\pi^{2} / 6-\gamma^{2}}{n}+\frac{(-10 / 3-2 \gamma) \ln n}{n} .
\end{aligned}
$$

Similarly, for $Y_{2}$, we obtain

$$
G(1, v)=\frac{v-4}{3(v-2)}+\frac{-v+4}{3 n}-\frac{2 n^{v} v}{\left(v^{2}-v-2\right) \Gamma(v) n^{2}},
$$

from which we derive

$$
\begin{aligned}
\mathbb{E}\left(Y_{2}\right) & \sim \frac{2}{3}+\frac{7 / 6+\gamma}{3 n}+\frac{\ln n}{n}, \\
\mathbb{V}\left(Y_{2}\right) & \sim \frac{14}{9}+\frac{8 \gamma / 3+19 / 9+\gamma^{2}-\pi^{2} / 6}{n}+\frac{(8 / 3+2 \gamma) \ln n}{n} .
\end{aligned}
$$

The covariance is now given by

$$
\operatorname{Cov}\left(Y_{1}, Y_{2}\right)=\frac{1}{9}-\frac{34}{9 n}+\mathcal{O}\left(\frac{\ln n}{n^{2}}\right)
$$


Of course, all these quantities can alternatively be obtained by suitable integrals again.

\section{INTERCHANGES}

In this final section, we want to compute the probability that two given elements $i$ and $j$ ever change places in Sattolo's and the Fisher-Yates algorithm respectively. These probabilities are deduced from recursive relations again - since the explicit formulæ have a very simple form, one can just guess them and verify them by means of induction. However, it is also possible to apply the techniques from the previous sections to these recursions. We start with the probabilities in Sattolo's algorithm:

Theorem 6.1. The probability that two elements $i \neq j$ are interchanged in Sattolo's algorithm is given by

$$
p_{n, i, j}=\frac{2(i+j+[i=1]+[j=1]-4)}{(n-1)(n-2)}= \begin{cases}\frac{2 i+2 j-8}{(n-1)(n-2)} & i, j>1, \\ \frac{2 i+2 j-6}{(n-1)(n-2)} & i=1 \text { or } j=1 .\end{cases}
$$

Proof. We consider two cases: if $i, j<n$, then there are two possibilities: it might happen that either $i$ or $j$ is interchanged with $n$ (with a probability of $\frac{1}{n-1}$ for each of these events). In either case, $i, j$ won't be interchanged. Otherwise, the probability that $i, j$ are interchanged is just $p_{n-1, i, j}$. Hence we have

$$
p_{n, i, j}=\frac{n-3}{n-1} p_{n-1, i, j}
$$

in this case. Now, we determine $p_{n, n, i}=p_{n, i, n}$. If $i, n$ are interchanged, the probability is clearly 1 . Otherwise, if $j, n(j<n, j \neq i)$ are interchanged, the probability is $p_{n-1, i, j}$. Thus we have

$$
p_{n, n, i}=\frac{1}{n-1}\left(1+\sum_{j=1}^{n-1} p_{n-1, i, j}\right)
$$

where we set $p_{n-1, i, i}=0$. Now, we proceed by induction. The formulæ are easily checked for $n=3$. We have, using the induction hypothesis,

$$
p_{n, i, j}=\frac{n-3}{n-1} \cdot \frac{2(i+j+[i=1]+[j=1]-4)}{(n-2)(n-3)}=\frac{2(i+j+[i=1]+[j=1]-4)}{(n-1)(n-2)}
$$

if $i, j<n$ and

$$
\begin{aligned}
p_{n, n, i} & =\frac{1}{n-1}\left(1+\sum_{\substack{j=1 \\
j \neq i}}^{n-1} \frac{2(i+j+[i=1]+[j=1]-4)}{(n-2)(n-3)}\right) \\
& =\frac{1}{(n-1)(n-2)(n-3)}\left((n-2)(n-3)+2(n-2)(i+[i=1]-4)+2 \sum_{\substack{j=1 \\
j \neq i}}^{n-1}(j+[j=1])\right) \\
& =\frac{1}{(n-1)(n-2)(n-3)}((n-2)(n+2 i+2[i=1]-11)+n(n-1)+2-2 i-2[i=1]) \\
& =\frac{2(n+i+[i=1]-4)}{(n-1)(n-2)},
\end{aligned}
$$

which finishes the proof.

A similar formula and proof can be given for interchanges in the Fisher-Yates algorithm: 
Theorem 6.2. The probability that two elements $i<j$ are interchanged in the Fisher-Yates algorithm is given by

$$
p_{n, i, j}=\frac{2 i+2 j-2 H_{i-1}-5}{n(n-1)} .
$$

Proof. Using the same arguments as in the previous proof, we have

$$
p_{n, i, j}=\frac{n-2}{n} p_{n-1, i, j}
$$

and

$$
p_{n, n, i}=\frac{1}{n}\left(1+\sum_{j=1}^{n-1} p_{n-1, i, j}\right),
$$

where we set $p_{n-1, i, i}=0$ again. The formula holds trivially for $n=2$, and we proceed by induction again. Then we obtain

$$
p_{n, i, j}=\frac{n-2}{n} \cdot \frac{2 i+2 j-2 H_{i-1}-5}{(n-1)(n-2)}=\frac{2 i+2 j-2 H_{i-1}-5}{n(n-1)}
$$

if $i, j<n$ and

$$
\begin{aligned}
p_{n, n, i} & =\frac{1}{n}\left(1+\sum_{j=1}^{i-1} \frac{2 i+2 j-2 H_{j-1}-5}{(n-1)(n-2)}+\sum_{j=i+1}^{n-1} \frac{2 i+2 j-2 H_{i-1}-5}{(n-1)(n-2)}\right) \\
& =\frac{1}{n(n-1)(n-2)}\left((n-1)(n-2)+\sum_{j=1}^{n-1}(2 i+2 j-5)-(4 i-5)-2 \sum_{j=1}^{i-1} H_{j-1}-2(n-i-1) H_{i-1}\right) \\
& =\frac{1}{n(n-1)(n-2)}\left(2 n^{2}-9 n+12+2 i(n-3)-2(i-1)\left(H_{i-1}-1\right)-2(n-i-1) H_{i-1}\right) \\
& =\frac{2 n+2 i-2 H_{i-1}-5}{n(n-1)},
\end{aligned}
$$

which finishes the proof again.

Note also that

$$
\sum_{1 \leq i<j \leq n} \frac{2(i+j+[i=1]+[j=1]-4)}{(n-1)(n-2)}=n-1
$$

(for Sattolo's algorithm) and

$$
\sum_{1 \leq i<j \leq n} \frac{2 i+2 j-2 H_{i-1}-5}{n(n-1)}=n-H_{n}
$$

(for the Fisher-Yates algorithm) give the correct values for the expected overall number of moves (neglecting moves which do not interchange two elements). Finally, one can also easily determine the probability that an element $i$ is interchanged with itself in the Fisher-Yates algorithm - here, we provide a more direct proof that doesn't depend on guessing the result first:

Theorem 6.3. The probability that an element $i$ is interchanged with itself at some point in the Fisher-Yates algorithm is given by

$$
p_{n, i}=\frac{H_{i-1}+1}{n} .
$$


Proof. It is plain that

$$
p_{n, i}= \begin{cases}\frac{n-1}{n} p_{n-1, i} & i<n, \\ \frac{1}{n}\left(1+\sum_{j=1}^{n-1} p_{n-1, j}\right) & i=n .\end{cases}
$$

Iterating the first equation, we find that $p_{n, i}=\frac{i p_{i, i}}{n}$. Plugging this into the second equation, we obtain

$$
p_{n, n}=\frac{1}{n}\left(1+\sum_{j=1}^{n-1} \frac{j p_{j, j}}{n-1}\right)
$$

or

$$
n(n-1) p_{n, n}=n-1+\sum_{j=1}^{n-1} j p_{j, j} .
$$

Taking differences yields

$$
n(n+1) p_{n+1, n+1}-n(n-1) p_{n, n}=1+n p_{n, n}
$$

or

$$
(n+1) p_{n+1, n+1}=n p_{n, n}+\frac{1}{n} .
$$

Since $p_{1,1}=1$, iterating yields $n p_{n, n}=H_{n-1}+1$ and thus $p_{n, i}=\frac{H_{i-1}+1}{n}$.

Let us finally remark that the average number of self-interchanges is thus

$$
\sum_{i=1}^{n} \frac{H_{i-1}+1}{n}=H_{n}
$$

as expected: $H_{n}$ is the expected number of times that the last element is chosen in the remaining file, as well as the expected number of cycles in a random permutation.

\section{FINAL REMARKS}

In principle, it seems possible to perform the same calculations for the parameter distance, i.e. the accumulated distance an element travels in Sattolo's or the Fisher-Yates algorithm. Indeed, one can easily provide the necessary recursions. In the case of Sattolo's algorithm, we have

$$
\phi_{n, k}^{(1)}(u)=\frac{u\left(u^{n-k}-1\right)}{(u-1)(n-1)}+\frac{k-1}{n-1} \phi_{k, k}(u)
$$

and

$$
\phi_{n, n}^{(1)}(u)=\frac{1}{n-1} \sum_{i=1}^{n-1} u^{n-i} \phi_{n-1, i}^{(1)}(u)
$$

for the movements of a single element and, for $1 \leq j<k<n$,

$$
\begin{aligned}
\phi_{n, k, j}^{(2)}(u, v) & =\frac{(k-1)(k-2)}{(n-1)(n-2)} \phi_{k, k, j}^{(2)}(u, v)+\sum_{l=1}^{n-k} \frac{n-l-1}{(n-1)(n-2)} u^{n-l-k+1} \phi_{n-l, j}^{(1)}(v) \\
& +\sum_{l=1}^{n-k} \frac{n-l-1}{(n-1)(n-2)} v^{n-l-j+1} \phi_{n-l, k}^{(1)}(u) .
\end{aligned}
$$


Finally, for $1 \leq j<n$, we have the recursion

$$
\phi_{n, n, j}^{(2)}(u, v)=\frac{1}{n-1}\left[\sum_{l=1}^{j-1} u^{n-l} \phi_{n-1, j, l}^{(2)}(v, u)+\sum_{l=j+1}^{n-1} u^{n-l} \phi_{n-1, l, j}^{(2)}(u, v)+v^{n-j} u^{n-j} \phi_{n-1, j}^{(1)}(u)\right] .
$$

Similar recursions can also be given for the Fisher-Yates algorithm; again, we start with the univariate generating functions:

$$
\phi_{n, k}^{(1)}(u)=\frac{u\left(u^{n-k}-1\right)}{(u-1) n}+\frac{k}{n} \phi_{k, k}(u)
$$

and

$$
\phi_{n, n}^{(1)}(u)=\frac{1}{n}\left(1+\sum_{i=1}^{n-1} u^{n-i} \phi_{n-1, i}^{(1)}(u)\right) .
$$

Furthermore, for $1 \leq j<k<n$,

$$
\begin{aligned}
\phi_{n, k, j}^{(2)}(u, v) & =\frac{k(k-1)}{n(n-1)} \phi_{k, k, j}^{(2)}(u, v)+\sum_{l=1}^{n-k} \frac{n-l}{n(n-1)} u^{n-l-k+1} \phi_{n-l, j}^{(1)}(v) \\
& +\sum_{l=1}^{n-k} \frac{n-l}{n(n-1)} v^{n-l-j+1} \phi_{n-l, k}^{(1)}(u) .
\end{aligned}
$$

Finally, for $1 \leq j<n$,

$\phi_{n, n, j}^{(2)}(u, v)=\frac{1}{n}\left[\sum_{l=1}^{j-1} u^{n-l} \phi_{n-1, j, l}^{(2)}(v, u)+\sum_{l=j+1}^{n-1} u^{n-l} \phi_{n-1, l, j}^{(2)}(u, v)+v^{n-j} u^{n-j} \phi_{n-1, j}^{(1)}(u)+u \phi_{n-1, j}^{(1)}(v)\right]$.

However, we refrain from analysing distances in the current paper, since the necessary computations are probably even longer and more intricate than the ones for the moves.

\section{REFERENCES}

[1] D. E. Knuth. The Art of Computer Programming, volume 2: Seminumerical Algorithms. Addison-Wesley, 1997. Third edition, 1997.

[2] Janice Lent and Hosam M. Mahmoud. Average-case analysis of multiple Quickselect: an algorithm for finding order statistics. Statist. Probab. Lett., 28(4):299-310, 1996.

[3] Guy Louchard and Helmut Prodinger. Random 0-1 rectangular matrices: a probabilistic analysis. Period. Math. Hungar., 47(1-2):169-193, 2003.

[4] Hosam M. Mahmoud. Mixed distributions in Sattolo's algorithm for cyclic permutations via randomization and derandomization. J. Appl. Probab., 40:790-796, 2003.

[5] Alois Panholzer and Helmut Prodinger. A generating functions approach for the analysis of grand averages for multiple QUICKSELECT. In Proceedings of the Eighth International Conference "Random Structures and Algorithms" (Poznan, 1997), volume 13, pages 189-209, 1998.

[6] Helmut Prodinger. Multiple Quickselect-Hoare's Find algorithm for several elements. Inform. Process. Lett., 56(3):123-129, 1995.

[7] Helmut Prodinger. On the analysis of an algorithm to generate a random cyclic permutation. Ars Combin., 65:75-78, 2002.

[8] Sandra Sattolo. An algorithm to generate a random cyclic permutation. Inform. Process. Lett., 22:315$317,1986$.

[9] Mark C. Wilson. Probability generating functions for Sattolos algorithm. J. Iranian Stat. Soc., 3:297-308, 2004

[10] Mark C. Wilson. Random and exhaustive generation of permutations and cycles. submitted, page 10 pages, 2006. 
Guy Louchard, Université Libre de Bruxelles, Département d’Informatique, CP 212, BouleVARd Du Triomphe, B-1050 Bruxelles, Belgium

E-mail address: louchard@ulb.ac.be

Helmut Prodinger, Department of Mathematics, University of Stellenbosch, 7602 StellenBOSCH, SOUTh AFricA

E-mail address: hproding@sun.ac.za

Stephan Wagner, Department of Mathematics, University of Stellenbosch, 7602 StellenBOSCH, South Africa

E-mail address: swagner@sun.ac.za 